\title{
One-Hour Contact with the Earth's Surface (Grounding) Improves Inflammation and Blood Flow-A Randomized, Double-Blind, Pilot Study
}

\author{
Gaétan Chevalier $^{1 *}$, Gregory Melvin ${ }^{2}$, Tiffany Barsotti ${ }^{3}$ \\ ${ }^{1}$ Developmental and Cell Biology Department, University of California at Irvine, Irvine, USA \\ ${ }^{2}$ Total Thermal Imaging, La Mesa, USA \\ ${ }^{3}$ California Institute for Human Science, Encinitas, USA \\ Email: ${ }^{\text {dlbogc@sbcglobal.net }}$
}

Received 23 July 2015; accepted 14 August 2015; published 17 August 2015

Copyright (C) 2015 by authors and Scientific Research Publishing Inc.

This work is licensed under the Creative Commons Attribution International License (CC BY). http://creativecommons.org/licenses/by/4.0/

\section{c) (i) Open Access}

\begin{abstract}
Earthing (grounding) refers to the human body being in contact with the surface of the Earth by barefoot exposure outdoors or using special indoor systems connected to the Earth. Previous studies have showed multiple beneficial effects as a result of such contact, including better sleep, normalization of cortisol, reduced inflammation, pain and stress, and better blood flow. To determine if Earthing for one hour improves facial blood circulation, forty middle-aged volunteers were divided into a grounded group and a sham-grounded group according to a double-blind procedure. They were asked to sit in a comfortable recliner chair equipped with a grounding mat, pillow and patches. The grounding systems were either grounded or sham-grounded via a wire to the ground port of a power outlet. An infrared imaging camera was used to measure changes in blood flow and temperature. Thermal imaging showed clearly improved circulation of fluids (including blood) throughout the torso, which in turn, translates into enhanced delivery of blood to the head and improved blood circulation in the face as well. The results of this innovative study demonstrate that even one-hour contact with the Earth appears to promote significantly autonomic nervous system control of body fluids and peripheral blood flow that may improve blood circulation in the torso and face, facial tissue repair, skin health and vitality and optimize facial appearance (face anterior view $p=0.002$; face lateral views $p=0.017$; full anterior torso view $p=0.002$ ). Further study using larger comparison groups and following subjects for a longer period of time (longitudinal study) is warranted.
\end{abstract}

\footnotetext{
${ }^{*}$ Corresponding author.
}

How to cite this paper: Chevalier, G., Melvin, G. and Barsotti, T. (2015) One-Hour Contact with the Earth's Surface (Grounding) Improves Inflammation and Blood Flow-A Randomized, Double-Blind, Pilot Study. Health, 7, 1022-1059. 


\section{Keywords}

\section{Earthing, Grounding, Thermal Imaging, Thermography, Blood Flow}

\section{Introduction}

Earthing (grounding, we use both terms interchangeably with the small difference that Earthing means grounding the body for the potential health benefits of being in contact with the Earth) is a practice whereby individuals walk barefoot outdoors and/or work, sleep, or relax indoors in contact with conductive mats, bed sheets, body bands, and patches that facilitate transfer of the Earth's surface charge into the body [1]. Unlike past cultures, most people today, particularly in industrial societies, rarely walk barefoot. They wear shoes with synthetic soles that insulate them from the Earth's natural electric charge, and no longer sleep on the ground. The Earth's negative surface charge (free electrons) is constantly replenished by the global atmospheric electrical circuit [2] [3]. The Earthing hypothesis states that when direct skin contact is made with the Earth's surface, an influx of electrons enters the body and equalizes the body's electric potential with that of the Earth [4] [5]. Published research on Earthing indicates that such contact yields a broad array of favorable health-related results including improved sleep, decreased pain, a normalizing effect on cortisol, reduction and/or normalization of stress, diminished damage to muscles caused by delayed onset muscle soreness (DOMS), reduction of primary indicators of osteoporosis, and improved glucose regulation, immune response and blood fluidity [6].

A study was conducted to determine if Earthing for one hour is beneficial to facial blood circulation. Two papers have already been published using data gathered during that study. The first publication presented results obtained with a laser speckle contrast imager (LSCI) to image blood flow and showed that Earthing improved facial blood flow and enhanced autonomic nervous system (ANS) regulation of peripheral circulation [7]. The second publication presented results showing that grounded subjects had significant improvement in mood while sham-grounded subjects did not show any improvement [8]. This time thermal imaging (TI) results are presented. The specific hypotheses related to TI are: 1) a statistically significant increase in skin temperature as measured by the TI camera when comparing thermal images of specific skin regions of the face, neck, and torso before and after the grounding period (no change is expected for the sham-grounding period); 2) significant improvements in skin temperature symmetry for the grounded group but not for the sham-grounded group; 3) a significant change in temperature relating to normalization of vascular/lymphatic activity bilaterally of $0.5^{\circ} \mathrm{C}$ or more of the same size parameter/geographical location taking into account inhibiting factors and organs involved with the lymphatic/venous return to the heart which include the diaphragm, gallbladder, and scalenus; 4) normalization (improved symmetry) about pain site parameter evaluation designated with a Pointer View (PV) performed by the subject. Confirmation of these hypotheses would suggest that grounding improves inflammation and blood circulation to the abdomen and face making it a natural way to rejuvenate facial skin and improving health.

\section{Methods}

\subsection{Bioethics Committee}

The protocol for this pilot study was approved by BioMed IRB of San Diego, California (http://www.biomedirb.com/) and was conducted at a single center: Total Thermal Imaging (TTI), La Mesa, California in 2014.

\subsection{Subjects}

Forty (40) subjects were recruited from the patient base of TTI. The number of subjects was determined based on the resources available. Subjects' average age and standard deviation (SD) were $54.8 \pm 9.8$ (grounded females $54.2 \pm 10.2$; grounded males $55.6 \pm 12.1$; sham-grounded females $58.7 \pm 6.5$; sham-grounded males 47.8 \pm 8.73). Subjects were randomly assigned to 2 groups: Group A, with 27 grounded individuals, and Group B, with 13 sham-grounded individuals (the control group). Sham-grounding means that subjects were going through the same grounding process as the grounding subjects but they were not actually grounded, the wire 
having been modified to not permit contact with the ground. Subjects were scheduled in the order they signed up to participate.

The exclusion criteria were: pregnancy, below the age of 18 or above 70, taking pain, anti-inflammatory medication, sedatives or prescription sleeping medication (less than 3 days prior to testing), taking psychotropic drugs or diagnosed with mental disorder, recent surgery (less than 3 months), documented life threatening disease (such as cancer, AIDS), consumption of alcohol within 48 hours of participation, smoking, use of recreational drugs, previous utilization of Earthing products or similar grounding products, going barefoot outdoors more than once a week and for more than half hour and not willing to comply with the Thermal Imaging Protocol Prior to Appointment (Appendix A).

\subsection{Earthing Equipment and Method}

The grounding equipment was provided by Earthing.com of Palm Springs, CA (www.earthing.com), which included conductive mats, pillows and transcutaneous electrical nerve stimulation (TENS) patches and wires. Subjects were grounded with the use of a grounding mat, grounding pillow and conductive patches connected via conducting wires into the ground port (third hole) of an electric power outlet after confirming that the TTI building had a proper and operational grounding system. The grounding wires contained a built-in $100 \mathrm{k} \Omega$ resistor for surge protection.

\subsection{Thermal Camera and Software}

TI, also known as thermography or infrared imaging, is a noninvasive neurovascular, autonomic, functional evaluation tool that measures skin temperature approximately $5 \mathrm{~mm}$ deep as a reflection of normal or abnormal human physiology. Skin is regulated primarily by the hypothalamus in the brain by way of the sympathetic nervous system (SNS). Every muscle, organ and gland blood supply is regulated by the hypothalamus with corresponding skin responses. TI demonstrates a functional autonomic evaluation of multiple systems at the same time, by reflecting the body parts as skin temperature differences. The technique utilizes sophisticated computerized technology to translate temperature data and produce an image that is then evaluated for signs of possible disease or injury. The procedure has been around for more than fifty years and used in thousands of medical studies. Among other things, TI is widely used to help assess breast cancer tumors, screen for diabetes, nervous system and metabolic disorders, soft tissue injuries, headaches and various complex pain syndromes, neck and back problems, and arterial disease [9] [10].

The data for this examination were acquired using the FLIR A310/A620, an infrared camera (FLIR Systems, International Main Office, Sweden, America's Main Office, USA Boston, MA. http://www.flir.com/US/) with a sensitivity of $0.01^{\circ} \mathrm{C}$ using appropriate software (Anthony Software, Thermography Unlimited LLC). The image resolution is $320 \times 240$ or $640 \times 480$ and the images were converted to PDF/JPEG format. The surface skin temperature of the following body areas was recorded and analyzed: face and neck (anterior and lateral views), and torso (anterior view only). The software allows to draw ellipses or rectangles on the image of any body area and to calculate the temperature average inside the ellipse or rectangle. These average temperatures are called "markers" and they can be placed anywhere on the image as an overlay. The markers used in this study and their location are presented in detail in Appendix B.

The software enables duplication of a marker anywhere by placing the mouse over the region of interest and by right clicking and then holding the button down. This way it is possible to position that marker to the opposite side of the body if portrayed on the same image. The overlays have 3 different colors to render a better visibility of the finding. The software has also the ability to transfer same size markers from one lateral image to the oppositional lateral view. This thermal imaging software is the only thermographic software allowing duplicate markers to be transferred to the opposing image.

With the continuous color palette used by the software (modified Rainbow Chrome) it has been found that optimal imaging to differentiate between colors is obtained with a $7^{\circ} \mathrm{C}$ temperature span (we will be using simply the word "span" to mean "temperature span" for the rest of this paper) between the coolest and warmest temperatures covered by the color palette. Span is defined as the number of degrees selected within a thermal image to best portray highest temperatures compared to the lowest temperatures while using a gray or color palette (Figure 1). Skin temperatures range from $20^{\circ} \mathrm{C}$ to $38^{\circ} \mathrm{C}$ thus positioning the temperature span above or below that range will render an all white or all back portrayal of that image. This can be seen clearly from Figure 2. 


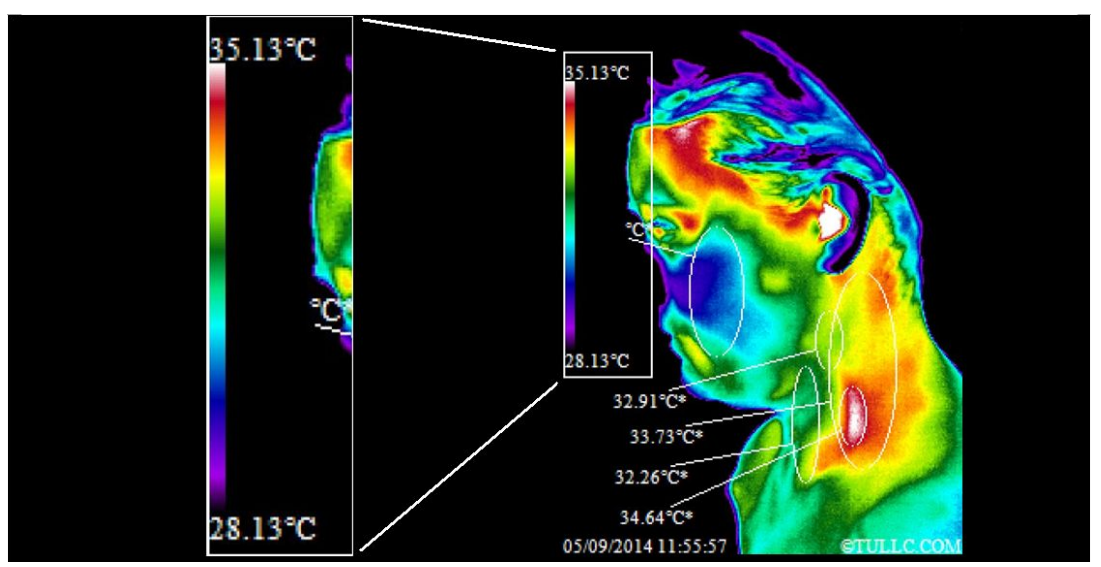

Figure 1. On the left side emphasis is put on the color distribution of the $7^{\circ} \mathrm{C}$ span according to the modified Rainbow Chrome color palette. In this case, the span is between $28.13^{\circ} \mathrm{C}$ and $35.13^{\circ} \mathrm{C}$. These maximum to minimum temperatures are adjusted to allow best view of color transitions on the areas of interest in the picture to the right (this is called span positioning).

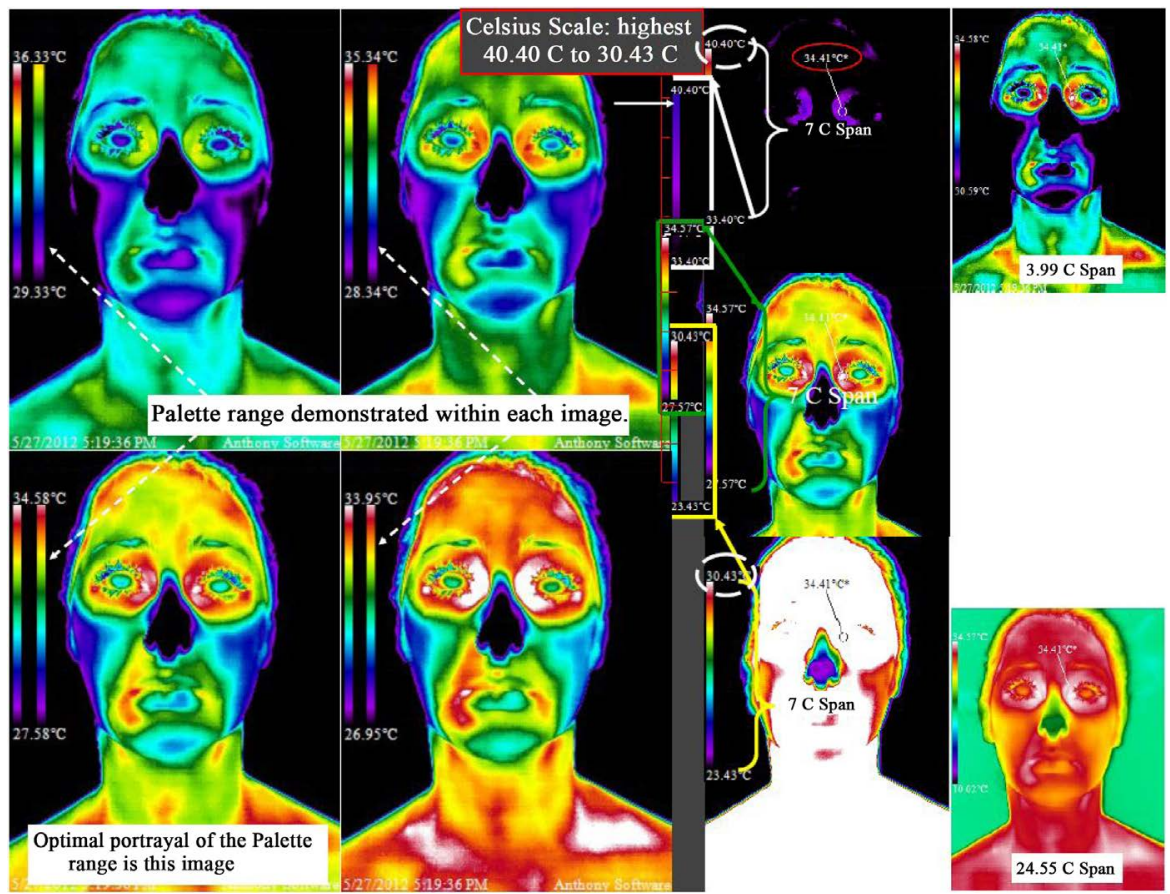

Figure 2. Visual effects of a range of temperatures called "span”, it's positioning and the color palette range. Except for the 2 small images on the right, all the images have a $7^{\circ} \mathrm{C}$ span. The middle image to the right of the 4 bigger images on the left has the optimal span positioning and color range as is the case for the image in the lower left corner (temperatures between $34.58^{\circ} \mathrm{C}$ and $27.58^{\circ} \mathrm{C} \pm 0.01^{\circ} \mathrm{C}$ ). The image on top of that middle image has its span positioned too high (between $40.40^{\circ} \mathrm{C}$ and $33.40^{\circ} \mathrm{C}$ ) making the subject almost blend with the dark background (looking too cold) while the image below it has its span positioned too low (between $30.43^{\circ} \mathrm{C}$ and $23.43^{\circ} \mathrm{C}$ ) making the subject appear extremely hot (almost all white, the warmest color). The 4 larger images on the left show how sensitive span positioning is and how it affects the range of colors (palette range) available in the picture. Between these 4 images the span positioning changed by only $2.38^{\circ} \mathrm{C}$ (hottest temperatures from $36.33^{\circ} \mathrm{C}$ and $33.95^{\circ} \mathrm{C}$; coldest temperatures from $29.33^{\circ} \mathrm{C}$ and $26.95^{\circ} \mathrm{C}$ ) and yet the effect is quite visible because the range of available colors from the full color palette (located to the left of the variable palette range) changed accordingly. The upper right image has a small span of $3.99^{\circ} \mathrm{C}$ making the subject look cold because the temperature transitions are too coarse. On the other hand, the image to the lower right shows what happens when the span is increased to $24.55^{\circ} \mathrm{C}$ : the subject looks almost all red (too hot) the temperature transitions are too small to show meaningful temperature variations. 
Color transitioning is more defined visually for transitions ranging from $0.3^{\circ} \mathrm{C}$ and above with a $7^{\circ} \mathrm{C}$ span. A larger span reduces visual recognition of temperature transitions while a smaller span may loose the context of certain regions of the body because of lack of contrast (Figure 2).

\subsection{Study Procedures}

Each subject was initially asked the questions contained in the TTI Wellness Entry Form (Appendix C) by one of the study coordinators to get basic health information and to make sure the subject was in compliance with this study's requirements. Next, the subject put on a cotton gown and was led to the TI room. The subject was then partially disrobed and underwent thermal equilibration for 15 minutes. The thermal examinations performed were consistent with recommended protocol for TI examinations and in a temperature-controlled environment with a constant temperature of $21.1^{\circ} \mathrm{C} \pm 0.6^{\circ} \mathrm{C}\left(70.0^{\circ} \mathrm{F} \pm 1.0^{\circ} \mathrm{F}\right)$ in a room with a minimum size of 2.44 $\times 2.44 \times 3.05 \mathrm{~m}^{3}(8 \times 8 \times 10$ cubic feet $)$ and with no drafts. Humidity was kept within $25 \%-50 \%$. Fluorescent lighting was used with covered windows to avoid solar infrared thermal artifacts on the images. While sitting on a stool, thermal images of the face, neck, and torso were taken, following the Thermal Imaging Protocol During Appointment described in Appendix A. This protocol was established based on the experience acquired with thermal imaging at TTI. Each subject identified areas of body pain by pointing at them. Pain areas have been identified with the letter "P" placed above them on the corresponding thermal images.

After TI was done, each subject moved to a comfortable recliner chair set up in the same room for the grounding (sham-grounding) session. The reclining angle of the chair was adjusted to a comfortable 30 degrees in respect to the plane of the floor. The chair back and seat were covered with a grounding mat. A grounding pillow was placed at the head position with a Styrofoam pad positioned under the pillow on each side to help stabilize the head and minimize movements. Patches were placed on both palms and soles (total of four patches). The connector ends of the wires from the patches, pillow and mat were inserted into the jacks of a connector box placed next to the recliner. A single wire connected the connector box to a ground port of an adjacent power outlet. A switch was installed on the wire from the connector box to the ground port to permit transference or blockage of the Earth's electrons (permitting or blocking conduction). This is schematically represented in Figure 3.

An initial ten minutes was dedicated to give time to subjects to relax. At end of that 10 -minute relaxation period, the switch was flipped on to permit grounding. However, the wires used for the sham-grounded controls were modified to not allow grounding independently of the position of the switch (on or off). After one hour, the grounding switch was turned off at the end of all sessions (grounded or sham-grounded). Each subject was then asked to return to the stool for a post-session round of TI.

A double-blind procedure prevented the researchers, study coordinators/technicians, and subjects from knowing whether an individual subject was actually grounded or sham-grounded. For that purpose, and to accommodate the fact that the design of the study called for about twice as many grounded subjects than sham-grounded subjects, three different colored-coded wires connecting patches, pillow and mat to the connector box were utilized. Wires with red and yellow tags permitted grounding; wires with the blue tag did not. The study coordinators randomly selected the wires' color for a particular session (wires to patches, pillow and mat had the same

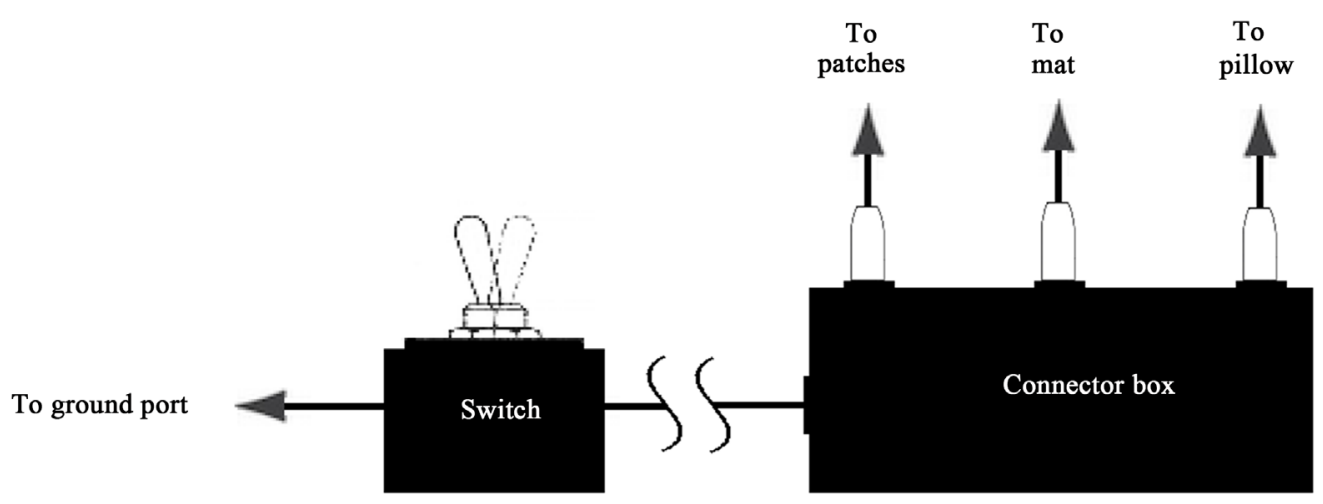

Figure 3. Grounding system comprising the connector box, including wires from patches, mat and pillow connected to it, with a wire going to a switch and then to the ground port of a power outlet. 
color for a particular session), and none of the study coordinators or subjects knew which color coded sham vs. functional wires.

\subsection{Statistical Analysis}

Statistical calculations were conducted using the NCSS/PASS 2000 Dawson edition statistical package. When all data used for a mean comparison were normally distributed (normality was observed) and the variances were substantially equal, t-test results are shown in the tables otherwise the results of nonparametric tests are presented. When the data were not normally distributed and/or when the variances were not substantially equal, the Wilcoxon Signed-Rank test (W S-R test) for difference in means with continuity correction was used to compare paired data and the Wilcoxon Rank-Sum test (W R-S test) for difference in means with correction was used for comparisons between groups. The usual value of $\alpha=0.05$ was applied to determine statistical significance. Two-tail distributions were assumed for all statistical tests. According to Uematsu [11], a change in temperature and/or in temperature asymmetry of $0.3^{\circ} \mathrm{C}$ or more is considered significant in TI. However, two groups reported that $0.5^{\circ} \mathrm{C}$ is better [12] [13], with both groups suggesting that the criterion of $1.0^{\circ} \mathrm{C}$ sometimes used would miss physiologically significant asymmetries. Therefore it was decided to use $0.5^{\circ} \mathrm{C}$ as the significant threshold to determine which temperature asymmetries or changes were significant.

\section{Results}

Varying individual responses of the subjects dictated presenting individual cases and space constrains to limit results presentation to three grounded subjects (A, B and C) and three sham-grounded (control) subjects (D, E and F) out of the 40 subjects who participated in the study. The results of these subjects were representative of the specific group results. For all six subjects, thermal images of three different views of the face (anterior, right lateral, and left lateral) and a full anterior view of the torso are shown. Subjects are presented in increasing age (younger to older) for each group.

\subsection{Thermal Images}

\subsubsection{Grounded Subjects}

1) Subject A-Female, Caucasian, 33, with history of extensive pain

In Figure 4, Subject A's facial thermal images were analyzed using a span of $7^{\circ} \mathrm{C}$ and positioning between $28.13^{\circ} \mathrm{C}$ and $35.13^{\circ} \mathrm{C}$. The color distribution, according to the modified Rainbow Chrome color palette, and the
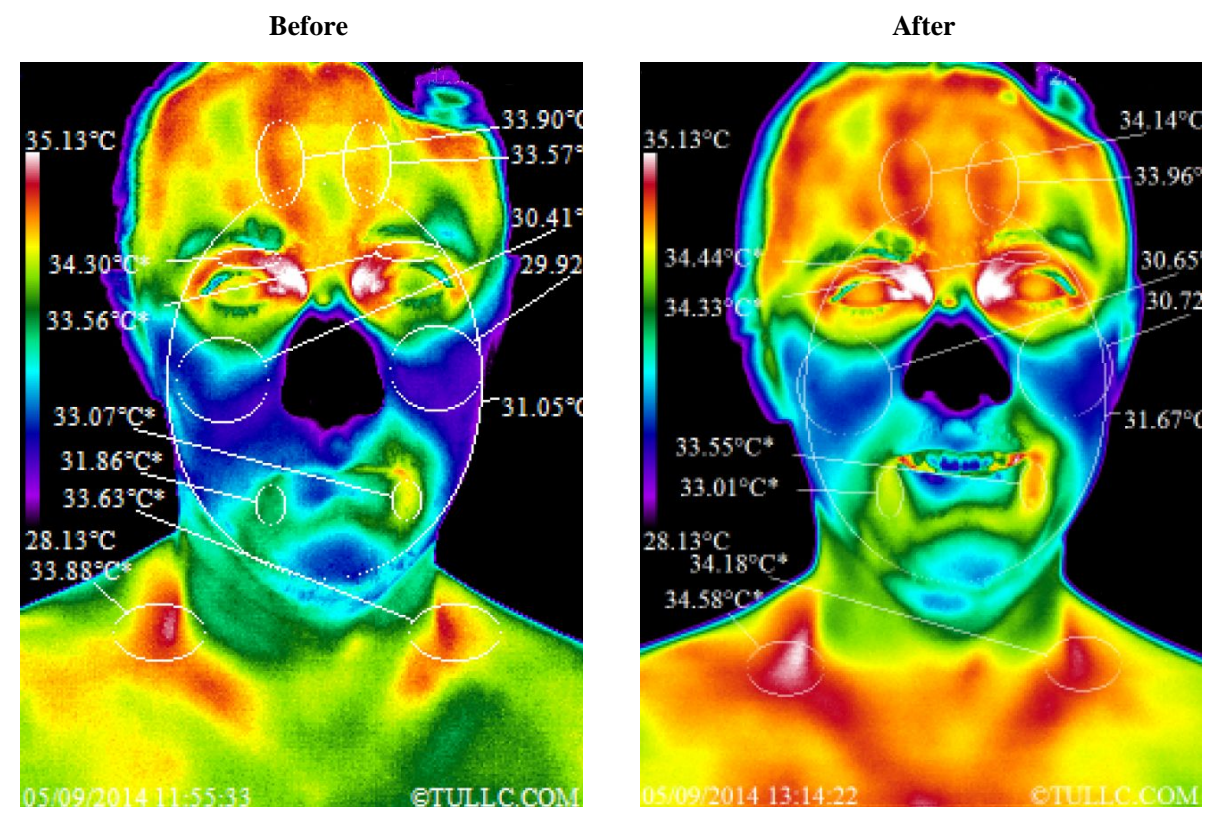

Figure 4. Subject A facial thermal images before and after grounding for one hour. 
$7^{\circ} \mathrm{C}$ span are displayed on the left side of each thermal image as explained previously (see Figure 1; this will be the case for all thermal images presented in this paper). After one hour of grounding, the temperature of the face increased significantly $\left(31.67^{\circ} \mathrm{C}-31.05^{\circ} \mathrm{C}=0.62^{\circ} \mathrm{C}\right)$. Statistical details of the image markers are presented in Table 1. Figure 5 top images show Subject A's right lateral side and bottom images show Subject A's left lateral side of the face before and after grounding. The temperature scale is the same as in Figure 4. Both lateral views reveal increased warmth throughout face and neck. Statistical details are presented in Table 2. Figure 6 shows full anterior views of Subject A's torso before and after one hour of grounding. The span for these images is between $27.40^{\circ} \mathrm{C}$ and $34.40^{\circ} \mathrm{C}$ for the left image and $27.39^{\circ} \mathrm{C}$ and $34.39^{\circ} \mathrm{C}$ for the right image. This difference in span positioning of $0.01^{\circ} \mathrm{C}$ (the sensitivity limit of the TI camera) between the before and after grounding images is small enough that it does not affect the comparison between the two images. For this subject, the entire anterior body clearly warmed up with the temperature of the region around the umbilicus showing a very significant increase $\left(34.35^{\circ} \mathrm{C}-31.84^{\circ} \mathrm{C}=2.51^{\circ} \mathrm{C}\right)$. Statistical details are presented in Table 4. For detail explanations of the significance of these temperature increases see the Discussion section.
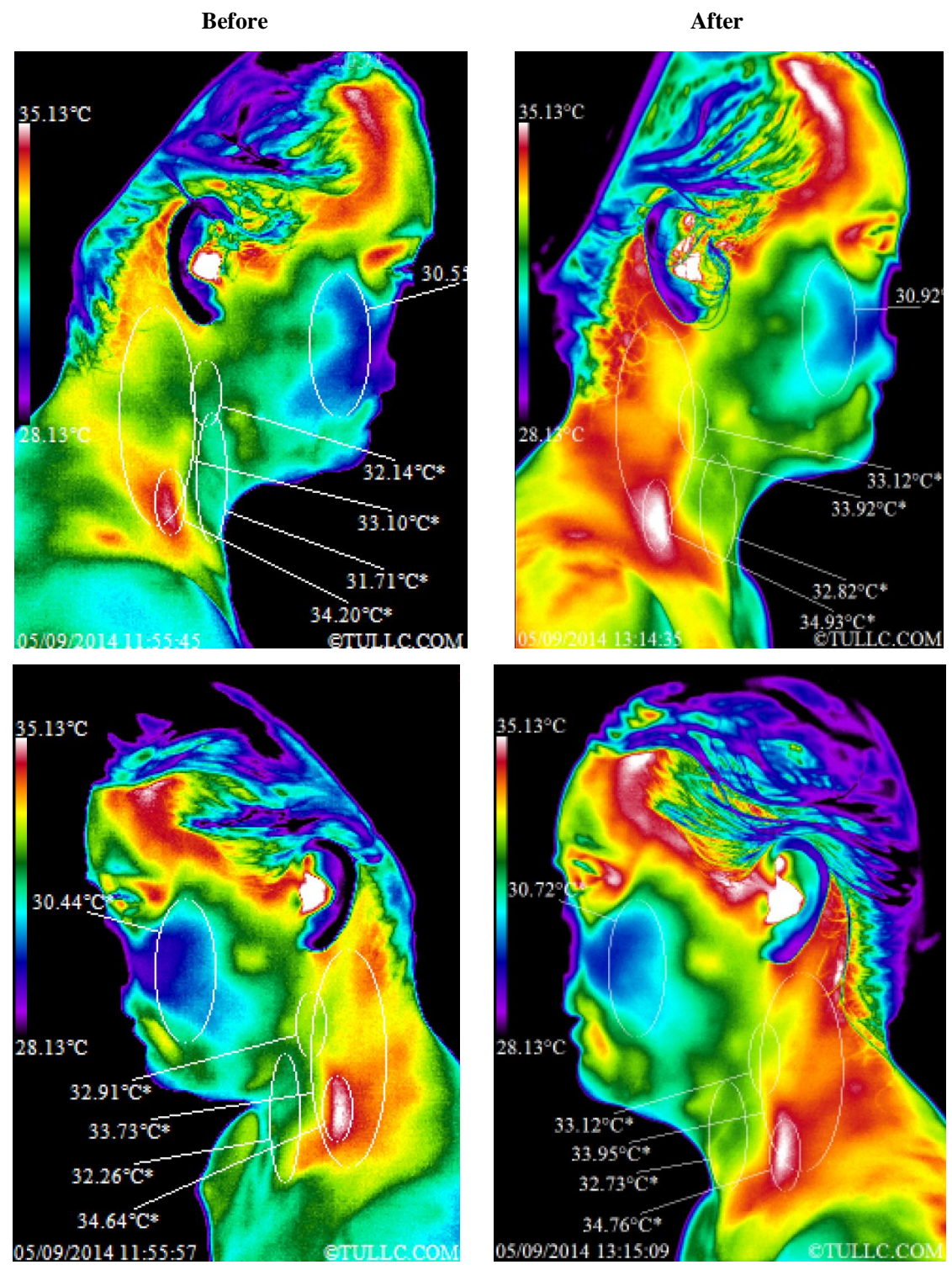

Figure 5. Top images: Subject A thermal images of the right lateral side of the face and neck before and after one hour of grounding. Bottom images: Subject A thermal images of the left lateral side of the face and neck before and after one hour of grounding. 

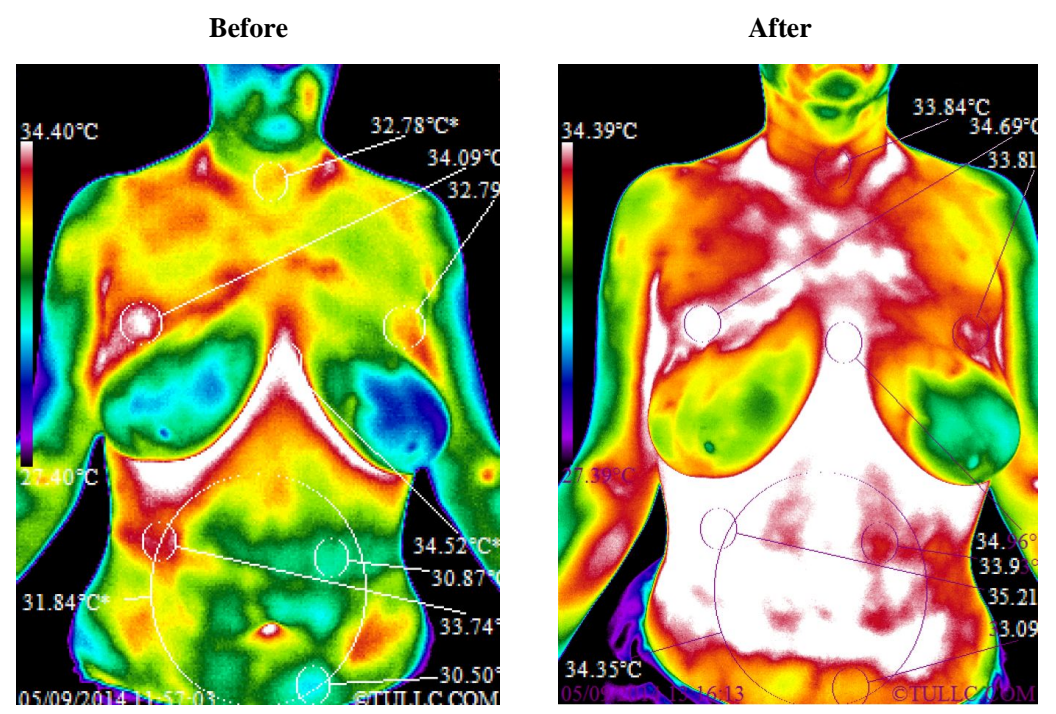

Figure 6. Subject A thermal images showing full anterior views of the torso before and after one hour of grounding.

Table 1. Face anterior view before vs. after group comparison statistics.

\begin{tabular}{|c|c|c|c|c|c|c|c|c|c|c|c|c|}
\hline \multirow{2}{*}{$\begin{array}{l}\text { Subject: } \\
\text { Marker }\end{array}$} & \multicolumn{3}{|c|}{ A } & \multicolumn{3}{|c|}{ B } & \multicolumn{3}{|c|}{ C } & \multicolumn{3}{|c|}{ Grounded Group Avg. } \\
\hline & Before & After & Diff & Before & After & Diff & Before & After & Diff & Before & After & Diff \\
\hline L Forehead & 33.57 & 33.96 & 0.39 & 34.20 & 33.57 & -0.63 & 33.11 & 32.41 & -0.70 & 33.63 & 33.31 & -0.31 \\
\hline L Eyelids & 33.56 & 34.33 & 0.77 & 34.89 & 33.84 & -1.05 & 33.58 & 32.63 & -0.95 & 34.01 & 33.60 & -0.41 \\
\hline L Cheeks & 29.92 & 30.72 & 0.80 & 30.94 & 30.37 & -0.57 & 31.00 & 30.41 & -0.59 & 30.62 & 30.50 & -0.12 \\
\hline L Mouth C. & 33.07 & 33.55 & 0.48 & 33.93 & 33.36 & -0.57 & 33.87 & 33.51 & -0.36 & 33.62 & 33.47 & -0.15 \\
\hline R Forehead & 33.90 & 34.14 & 0.24 & 34.30 & 33.70 & -0.60 & 33.00 & 32.31 & -0.69 & 33.73 & 33.38 & -0.35 \\
\hline R Eyelids & 34.30 & 34.44 & 0.14 & 35.09 & 34.25 & -0.84 & 33.50 & 33.20 & -0.30 & 34.30 & 33.96 & -0.33 \\
\hline R Cheeks & 30.41 & 30.65 & 0.24 & 30.88 & 30.60 & -0.28 & 31.31 & 29.92 & -1.39 & 30.87 & 30.39 & -0.48 \\
\hline R Mouth C. & 31.86 & 33.01 & 1.15 & 34.70 & 34.33 & -0.37 & 33.66 & 33.33 & -0.33 & 33.41 & 33.56 & 0.15 \\
\hline Average: & 32.57 & 33.10 & 0.53 & 33.62 & 33.00 & -0.61 & 32.88 & 32.22 & -0.66 & 33.02 & 32.77 & -0.25 \\
\hline SD: & 1.66 & 1.56 & -0.10 & 1.71 & 1.59 & -0.12 & 1.10 & 1.34 & 0.24 & 1.43 & 1.45 & 0.02 \\
\hline Normality: & Yes & No & Yes & No & No & Yes & No & Yes & Yes & No & No & Yes \\
\hline W S-R test: & \multicolumn{3}{|c|}{0.0143} & \multicolumn{3}{|c|}{0.0141} & \multicolumn{2}{|c|}{0.0143} & \multicolumn{4}{|c|}{0.0423} \\
\hline Subject: & \multicolumn{3}{|c|}{ D } & \multicolumn{3}{|c|}{$\mathbf{E}$} & \multicolumn{3}{|c|}{$\mathbf{F}$} & \multicolumn{3}{|c|}{ Control Group Avg. } \\
\hline Marker & Before & After & Diff & Before & After & Diff & Before & After & Diff & Before & After & Diff \\
\hline L Forehead & 34.16 & 34.01 & -0.15 & 32.94 & 32.21 & -0.73 & 32.26 & 31.75 & -0.51 & 33.12 & 32.66 & -0.46 \\
\hline L Eyelids & 35.72 & 35.51 & -0.21 & 33.93 & 33.30 & -0.63 & 33.53 & 32.93 & -0.60 & 34.39 & 33.91 & -0.48 \\
\hline L Cheeks & 33.03 & 32.13 & -0.90 & 30.90 & 29.98 & -0.92 & 30.84 & 29.61 & -1.23 & 31.59 & 30.57 & -1.02 \\
\hline L Mouth C. & 35.43 & 34.86 & -0.57 & 33.84 & 32.83 & -1.01 & 32.44 & 32.55 & 0.11 & 33.90 & 33.41 & -0.49 \\
\hline R Forehead & 34.65 & 34.44 & -0.21 & 32.70 & 31.91 & -0.79 & 32.23 & 31.79 & -0.44 & 33.19 & 32.71 & -0.48 \\
\hline R Eyelids & 35.99 & 35.62 & -0.37 & 33.55 & 33.14 & -0.41 & 33.68 & 32.69 & -0.99 & 34.41 & 33.82 & -0.59 \\
\hline R Cheeks & 33.47 & 32.38 & -1.09 & 31.01 & 30.01 & -1.00 & 31.09 & 30.31 & -0.78 & 31.86 & 30.90 & -0.96 \\
\hline R Mouth C. & 35.03 & 34.21 & -0.82 & 34.37 & 33.58 & -0.79 & 33.87 & 33.54 & -0.33 & 34.42 & 33.78 & -0.65 \\
\hline Average: & 34.69 & 34.15 & -0.54 & 32.91 & 32.12 & -0.79 & 32.49 & 31.90 & -0.60 & 33.36 & 32.72 & -0.64 \\
\hline SD: & 1.07 & 1.30 & 0.24 & 1.32 & 1.42 & 0.10 & 1.15 & 1.34 & 0.19 & 1.14 & 1.32 & 0.18 \\
\hline t-test: & \multicolumn{2}{|c|}{0.00386} & \multicolumn{4}{|c|}{0.00001} & \multicolumn{2}{|c|}{0.00465} & \multicolumn{4}{|c|}{0.00008} \\
\hline Normality: & Yes & Yes & Yes & Yes & Yes & Yes & Yes & Yes & Yes & Yes & Yes & No \\
\hline W R-S test: & & & & & & & & & & 0.564 & 0.958 & 0.002 \\
\hline
\end{tabular}

L = Left; $\mathrm{R}=$ Right; C. = Corner. 
Table 2. Face right and left lateral views before vs. after comparison statistics.

\begin{tabular}{|c|c|c|c|c|c|c|c|c|c|c|c|c|}
\hline \multirow{2}{*}{$\begin{array}{l}\text { Subject: } \\
\text { Marker }\end{array}$} & \multicolumn{3}{|c|}{ A } & \multicolumn{3}{|c|}{ B } & \multicolumn{3}{|c|}{ C } & \multicolumn{3}{|c|}{ Grounded Group Avg. } \\
\hline & Before & After & Diff & Before & After & Diff & Before & After & Diff & Before & After & Diff \\
\hline L Cheeks & 30.44 & 30.72 & 0.28 & 31.28 & 30.57 & -0.71 & 31.64 & 30.81 & -0.83 & 31.12 & 30.70 & -0.42 \\
\hline L Carotid Bif. & 32.91 & 33.12 & 0.21 & 33.89 & 33.65 & -0.24 & 33.61 & 33.21 & -0.40 & 33.47 & 33.33 & -0.14 \\
\hline L Back Neck & 33.73 & 33.95 & 0.22 & 33.94 & 33.76 & -0.18 & 33.79 & 33.75 & -0.04 & 33.82 & 33.82 & 0.00 \\
\hline L Front Neck & 32.26 & 32.73 & 0.47 & 33.36 & 33.01 & -0.35 & 32.51 & 32.59 & 0.08 & 32.71 & 32.78 & 0.07 \\
\hline L Supraclav. & 34.64 & 34.76 & 0.12 & 34.61 & 34.22 & -0.39 & 33.61 & 33.80 & 0.19 & 34.29 & 34.26 & -0.03 \\
\hline R Cheeks & 30.55 & 30.92 & 0.37 & 31.91 & 30.34 & -1.57 & 31.14 & 30.12 & -1.02 & 31.20 & 30.46 & -0.74 \\
\hline R Carotid bif. & 32.14 & 33.12 & 0.98 & 34.08 & 33.97 & -0.11 & 33.50 & 33.25 & -0.25 & 33.24 & 33.45 & 0.21 \\
\hline R Back Neck & 33.10 & 33.92 & 0.82 & 34.00 & 33.93 & -0.07 & 33.67 & 34.15 & 0.48 & 33.59 & 34.00 & 0.41 \\
\hline R Front Neck & 31.71 & 32.82 & 1.11 & 33.27 & 32.56 & -0.71 & 32.54 & 32.60 & 0.06 & 32.51 & 32.66 & 0.15 \\
\hline R Supraclav. & 34.20 & 34.93 & 0.73 & 34.24 & 33.99 & -0.25 & 33.66 & 33.61 & -0.05 & 34.03 & 34.18 & 0.14 \\
\hline Average: & 32.57 & 33.10 & 0.53 & 33.46 & 33.00 & -0.46 & 32.97 & 32.79 & -0.18 & 33.00 & 32.96 & -0.04 \\
\hline SD: & 1.43 & 1.42 & -0.01 & 1.07 & 1.43 & 0.37 & 0.96 & 1.33 & 0.37 & 1.11 & 1.37 & 0.26 \\
\hline t-test: & \multicolumn{2}{|c|}{0.00103} & & & & & & & & & & \\
\hline Normality: & Yes & Yes & Yes & No & No & No & No & No & Yes & Yes & No & Yes \\
\hline W S-R test: & & & & \multicolumn{2}{|c|}{0.00589} & & \multicolumn{2}{|c|}{0.476} & \multicolumn{4}{|c|}{0.878} \\
\hline Subject: & \multicolumn{3}{|c|}{ D } & \multicolumn{3}{|c|}{$\mathbf{E}$} & \multicolumn{3}{|c|}{$\mathbf{F}$} & \multicolumn{3}{|c|}{ Control Group Avg. } \\
\hline Marker & Before & After & Diff & Before & After & Diff & Before & After & Diff & Before & After & Diff \\
\hline L Cheeks & 33.42 & 32.61 & -0.81 & 31.28 & 30.58 & -0.70 & 31.63 & 30.41 & -1.22 & 32.11 & 31.20 & -0.91 \\
\hline L Carotid Bif. & 34.33 & 33.37 & -0.96 & 32.67 & 32.94 & 0.27 & 34.02 & 32.89 & -1.13 & 33.67 & 33.07 & -0.61 \\
\hline L Back Neck & 34.25 & 33.63 & -0.62 & 33.32 & 33.94 & 0.62 & 34.43 & 34.09 & -0.34 & 34.00 & 33.89 & -0.11 \\
\hline L Front Neck & 34.06 & 33.42 & -0.64 & 32.65 & 32.80 & 0.15 & 33.31 & 33.10 & -0.21 & 33.34 & 33.11 & -0.23 \\
\hline L Supraclav. & 34.05 & 33.75 & -0.30 & 34.46 & 34.79 & 0.33 & 35.11 & 35.22 & 0.11 & 34.54 & 34.59 & 0.05 \\
\hline R Cheeks & 33.30 & 32.45 & -0.85 & 31.37 & 30.31 & -1.06 & 31.68 & 30.89 & -0.79 & 32.12 & 31.22 & -0.90 \\
\hline R Carotid bif. & 35.03 & 34.51 & -0.52 & 33.07 & 33.30 & 0.23 & 33.84 & 33.36 & -0.48 & 33.98 & 33.72 & -0.26 \\
\hline R Back Neck & 34.62 & 33.87 & -0.75 & 33.32 & 33.91 & 0.59 & 34.18 & 32.91 & -1.27 & 34.04 & 33.56 & -0.48 \\
\hline R Front Neck & 34.08 & 33.24 & -0.84 & 32.60 & 32.79 & 0.19 & 33.19 & 32.28 & -0.91 & 33.29 & 32.77 & -0.52 \\
\hline R Supraclav. & 35.13 & 34.37 & -0.76 & 34.16 & 34.14 & -0.02 & 34.88 & 34.98 & 0.10 & 34.72 & 34.50 & -0.23 \\
\hline Average: & 34.23 & 33.52 & -0.70 & 32.89 & 32.95 & 0.06 & 33.63 & 33.01 & -0.61 & 33.58 & 33.16 & -0.42 \\
\hline SD: & 0.60 & 0.66 & 0.07 & 1.03 & 1.47 & 0.44 & 1.20 & 1.56 & 0.36 & 0.90 & 1.18 & 0.29 \\
\hline t-test: & \multicolumn{2}{|c|}{0.000001} & \multicolumn{4}{|c|}{0.732} & \multicolumn{2}{|c|}{0.00489} & \multicolumn{4}{|c|}{0.00256} \\
\hline Normality: & Yes & Yes & Yes & Yes & Yes & No & Yes & Yes & Yes & Yes & Yes & Yes \\
\hline t-test: & & & & & & & & & & 0.213 & & 0.017 \\
\hline Normality: & & & & & & & & & & Yes & Yes & Yes \\
\hline Equal Variance: & & & & & & & & & & Yes & Yes & Yes \\
\hline W R-S test: & & & & & & & & & & & 0.791 & \\
\hline
\end{tabular}

L = Left; R = Right; Supraclav. = Supraclavicular; Bif. = Bifurcation. 
2) Subject B-Female, Caucasian, 49, with history of pain and surgery for right thumb and right lateral breast region

Subject B's facial thermal images were analyzed using a span of $7.01^{\circ} \mathrm{C}$ and positioning between $28.22^{\circ} \mathrm{C}$ and $35.23^{\circ} \mathrm{C}$. Figure 7 shows that the temperature of her face decreased significantly $\left(32.54^{\circ} \mathrm{C}-31.95^{\circ} \mathrm{C}=0.59^{\circ} \mathrm{C}\right)$. Statistical details of the image markers are presented in Table 1. Figure 8 top images show Subject B's right
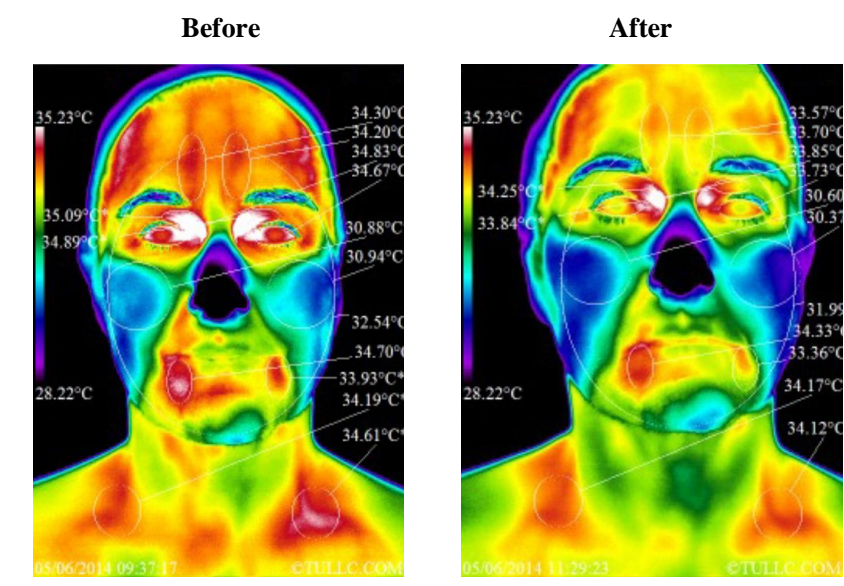

Figure 7. Subject B facial thermal images before and after one hour of grounding.

Before
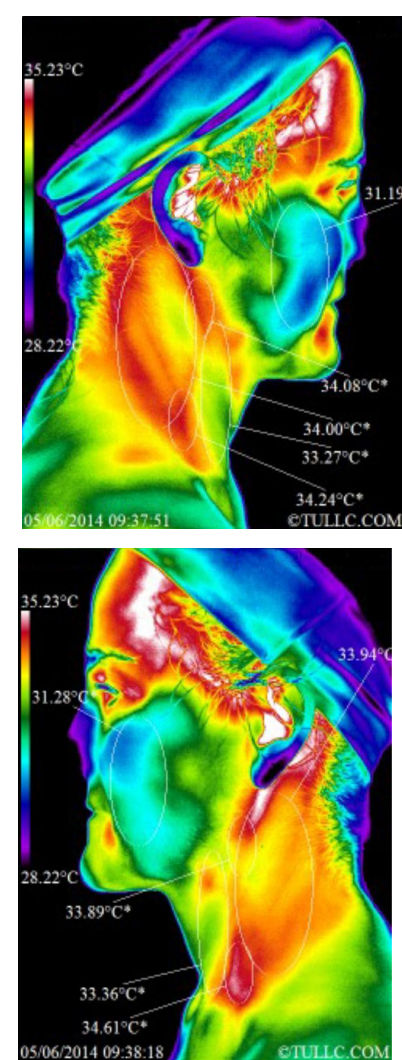

After
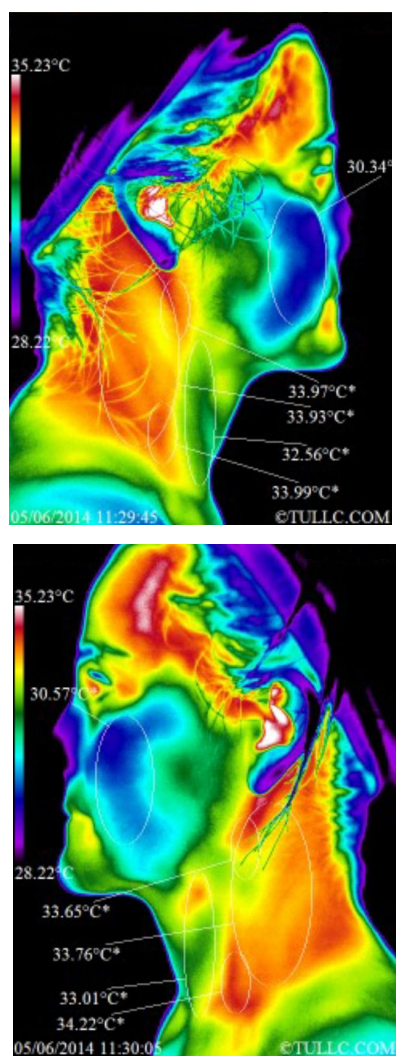

Figure 8. Top images: Subject B thermal images of the right lateral side of the face and neck before and after one hour of grounding. Bottom images: Subject B thermal images of the left lateral side of the face and neck before and after one hour of grounding. 
lateral views of the face and the images below those show left lateral views of the face before and after the grounding session. The span and positioning is the same as in Figure 7. Both sides of the face clearly cooled off. For example the marker of the right cheek decreased significantly $\left(31.19{ }^{\circ} \mathrm{C}-30.34^{\circ} \mathrm{C}=0.85^{\circ} \mathrm{C}\right)$. Statistical details are presented in Table 2. Figure 9 shows full anterior views of the torso before and after the grounding session. This subject pointed a region of pain on the right side of the right breast near the arm pit, as shown by the circled letter "P" in the before picture of Figure 9. The span and positioning for these images is between $27.54^{\circ} \mathrm{C}$ and $34.55^{\circ} \mathrm{C}$. The temperature of the abdomen increased but the temperature around the neck and shoulders decreased. Also the temperature of the right breast decreased while that of the left breast increased bringing them into an improved temperature symmetry. The temperature of the region around the umbilicus increased by exactly $0.5^{\circ} \mathrm{C}\left(32.74^{\circ} \mathrm{C}-32.24^{\circ} \mathrm{C}=0.5^{\circ} \mathrm{C}\right)$. Statistical details are presented in Table 4 and interpretation of patterns in the Discussion section.

3) Subject C-Female, Caucasian, 55, with history of uterine fibroids and lower pelvic pain

Subject C's facial thermal images were analyzed using a span of $7.01^{\circ} \mathrm{C}$ and positioning between $27.65^{\circ} \mathrm{C}$ and $34.66^{\circ} \mathrm{C}$. Figure 10 shows that the temperature of her face decreased significantly $\left(32.28^{\circ} \mathrm{C}-31.51^{\circ} \mathrm{C}=0.77^{\circ} \mathrm{C}\right)$. The temperature of the supraclavicular regions did not change significantly and there is no significant change in
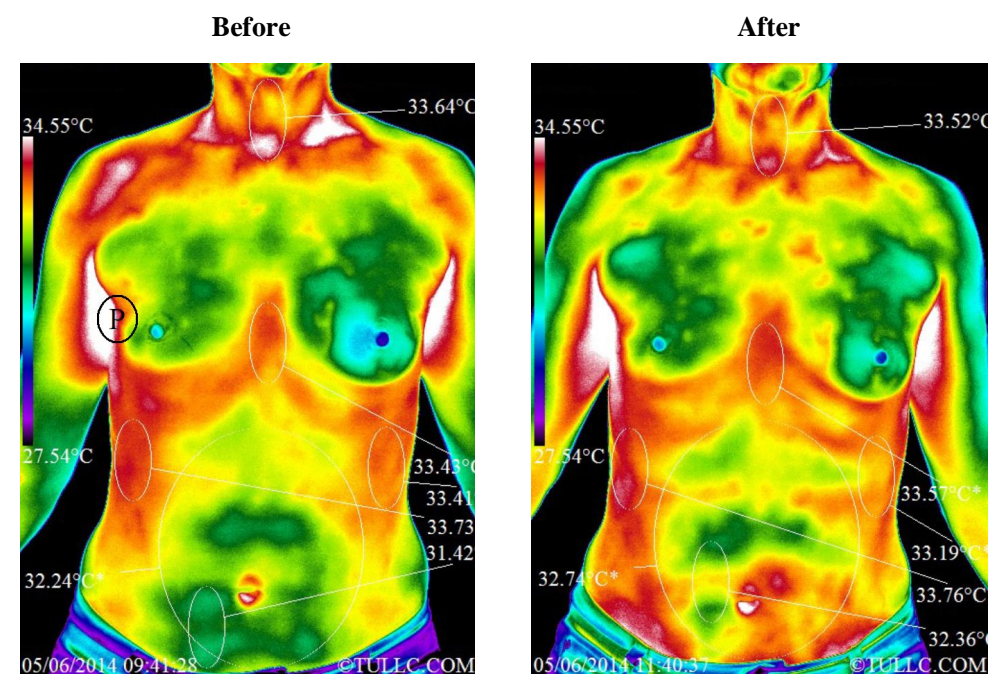

Figure 9. Subject B thermal images showing full anterior views of the torso before and after one hour of grounding.
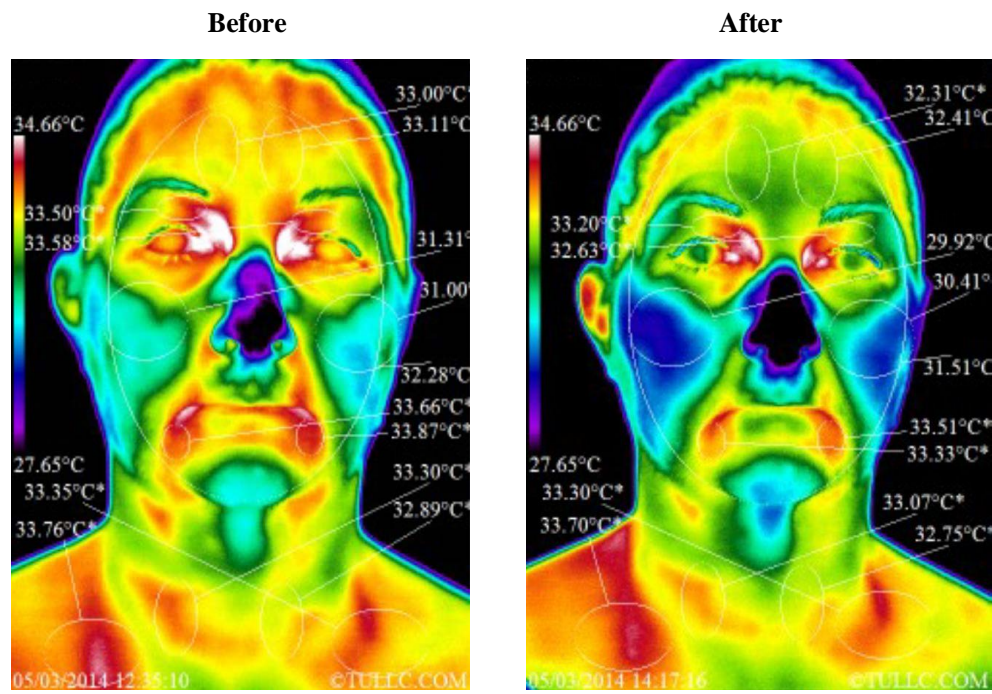

Figure 10. Subject C facial thermal images before and after one hour of grounding. 
the asymmetry. Statistical details of the image markers are presented in Table 1. Figure 11 top images show Subject C's right lateral side of the face and the images below show her facial left lateral side before and after the grounding session. The span and positioning is the same as in Figure 10. The right lateral side of the face (particularly the cheek) and the anterior neck cooled off while the posterior lateral aspects of her neck became warmer. On the left lateral side, we see a similar pattern i.e. the anterior neck cooled off while the posterior lateral aspects of the neck and the supraclavicular region warmed up. Statistical details are presented in Table 2. Figure 12 shows full anterior views of Subject C's torso before and after the grounding session. The span and positioning for these images is between $27.77^{\circ} \mathrm{C}$ and $34.78^{\circ} \mathrm{C}$. The regions marked with the letter "P" on the left thermal image are regions of pain as pointed out by the subject. The temperature of the torso in general decreased while the temperature of the region around the umbilicus increased but not significantly except for the region immediately above the umbilicus $\left(32.12^{\circ} \mathrm{C}-30.87^{\circ} \mathrm{C}=1.25^{\circ} \mathrm{C}\right)$. Statistical details are presented in Table 4.
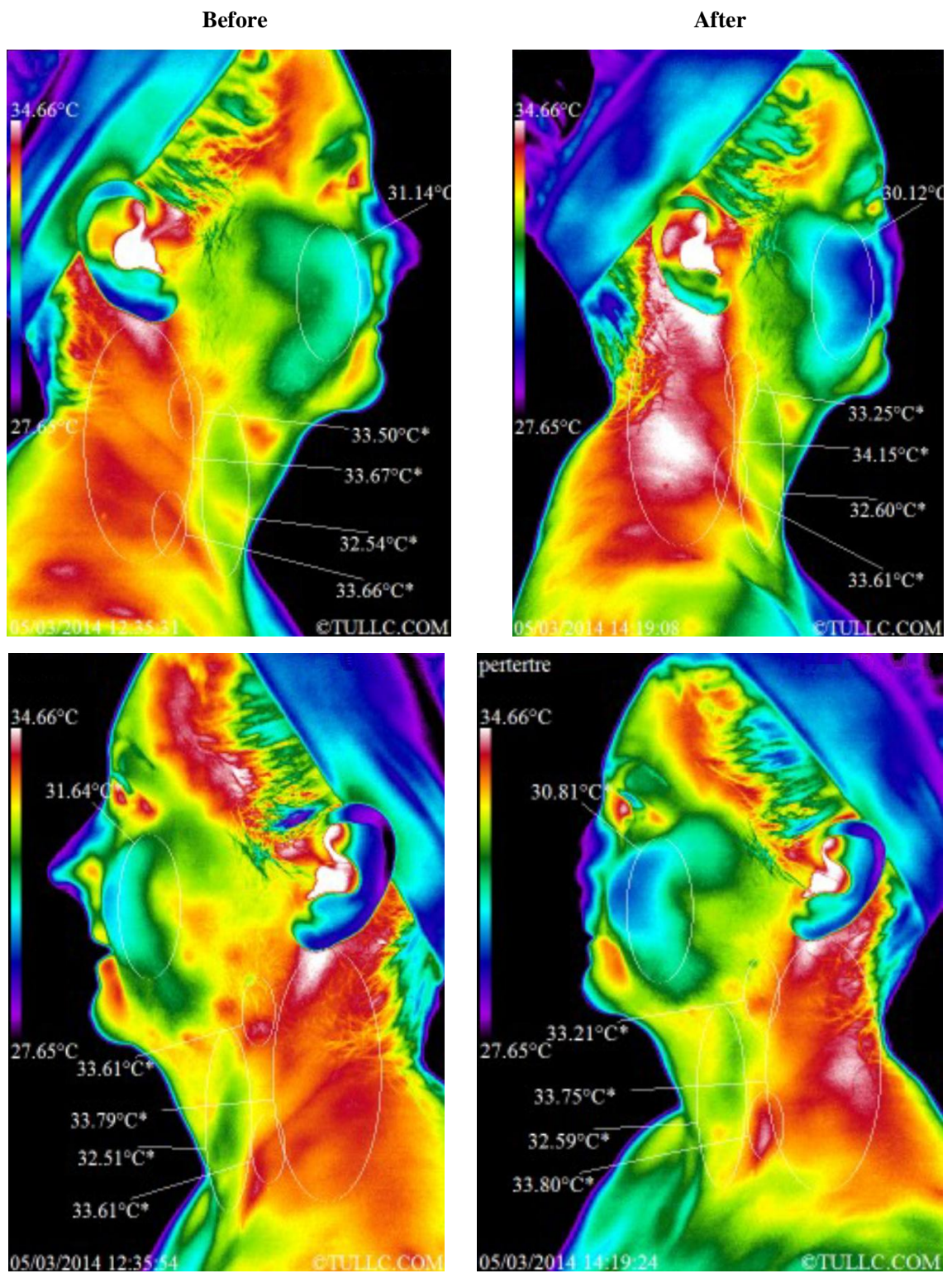

Figure 11. Top images: Subject $C$ thermal images of the right lateral side of the face and neck before and after one hour of grounding. Bottom images: Subject $\mathrm{C}$ thermal images of the left lateral side of the face and neck before and after one hour of grounding. 

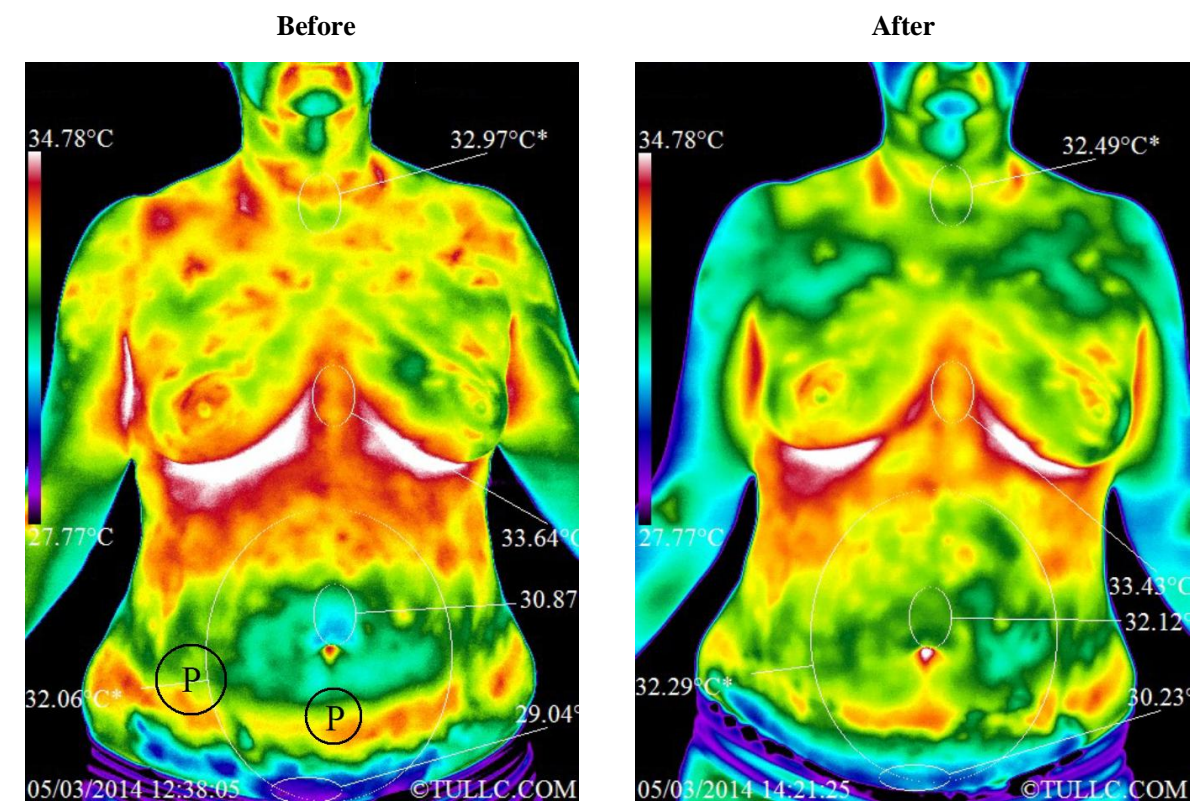

Figure 12. Subject $\mathrm{C}$ thermal images showing full anterior views of the torso before and after one hour of grounding.

\subsubsection{Sham-Grounded (Control) Subjects}

1) Subject D-Male, Caucasian, 42, with bulging disc and left lateral foot numbness

Subject D's facial thermal images were analyzed using a span of $7^{\circ} \mathrm{C}$ and positioning between $29.13^{\circ} \mathrm{C}$ and $36.13^{\circ} \mathrm{C}$. In Figure 13, the temperature of the face decreased significantly $\left(34.44^{\circ} \mathrm{C}-33.68^{\circ} \mathrm{C}=0.76^{\circ} \mathrm{C}\right)$. The temperature of the supraclavicular region on the right lateral side decreased by exactly $1^{\circ} \mathrm{C}\left(35.07^{\circ} \mathrm{C}-34.07^{\circ} \mathrm{C}=\right.$ $\left.1.00^{\circ} \mathrm{C}\right)$ while the supraclavicular region on the left lateral side also decreased by more than $0.5^{\circ} \mathrm{C}\left(34.35^{\circ} \mathrm{C}-\right.$ $33.61^{\circ} \mathrm{C}=0.74^{\circ} \mathrm{C}$ ). The asymmetry of this region was significant before the sham-grounding session and decreased after but not significantly (before $35.07^{\circ} \mathrm{C}-34.35^{\circ} \mathrm{C}=0.72^{\circ} \mathrm{C}$; after $34.07^{\circ} \mathrm{C}-33.61^{\circ} \mathrm{C}=0.46^{\circ} \mathrm{C}$, a decrease by $0.26^{\circ} \mathrm{C}$ ). Statistical details of the image markers are presented in Table 1. Figure 14 top images show Subject D right lateral side and bottom images show left lateral side of the face before and after a one hour sham-grounding session. Thermal images reveal that both right and left sides of the face and neck decreased significantly in temperature after the sham-grounding session. Statistical details are presented in Table 2. Figure 15 shows full anterior views of Subject D's torso before and after a one hour sham-grounding session. The span and positioning for these images is between $28.37^{\circ} \mathrm{C}$ and $35.37^{\circ} \mathrm{C}$. The entire anterior torso cooled off significantly with the exception of the left axilla which seemed to have warmed up slightly. The temperature of the region around the umbilicus decreased very significantly $\left(32.26^{\circ} \mathrm{C}-30.02^{\circ} \mathrm{C}=2.24^{\circ} \mathrm{C}\right)$. Statistical details are presented in Table 4.

2) Subject E-Female, Caucasian, 55, with bilateral pelvic pain and inner right arm pain from shoulder to hand

Subject E's facial thermal images were analyzed using a span of $7.01^{\circ} \mathrm{C}$ and positioning between $27.76^{\circ} \mathrm{C}$ and $34.77^{\circ} \mathrm{C}$. Figure 16 shows that the temperature of the face decreased by a very significant $1.32^{\circ} \mathrm{C}\left(32.75^{\circ} \mathrm{C}\right.$ $31.43^{\circ} \mathrm{C}=1.32^{\circ} \mathrm{C}$ ). The temperature of the supraclavicular regions did not change significantly, and the asymmetry of these regions decreased but not significantly. Statistical details of the image markers are presented in Table 1. Figure 17 top images show right lateral side and bottom images show left lateral side of the face before and after the sham-grounding session. Here, the thermal images reveal that the cheeks on both sides cooled off significantly (right side: $31.37^{\circ} \mathrm{C}-30.31^{\circ} \mathrm{C}=1.06^{\circ} \mathrm{C}$; left side: $31.28^{\circ} \mathrm{C}-30.58^{\circ} \mathrm{C}=0.70^{\circ} \mathrm{C}$ ) while the neck became significantly warmer (right side: $33.91^{\circ} \mathrm{C}-33.32^{\circ} \mathrm{C}=0.59^{\circ} \mathrm{C}$; left side: $33.94^{\circ} \mathrm{C}-33.32^{\circ} \mathrm{C}=0.62^{\circ} \mathrm{C}$ ). Note also that the temperature of the right supraclavicular region remained about the same but the left supraclavicular region warmed up, although not significantly. Statistical details are presented in Table 2. Subject E's full anterior views of the torso are presented in Figure 18. The subject noted pelvic pain on both sides of the abdomen and pain in the inner side of the right arm and shoulder. They are indicated in the left thermal image by the 

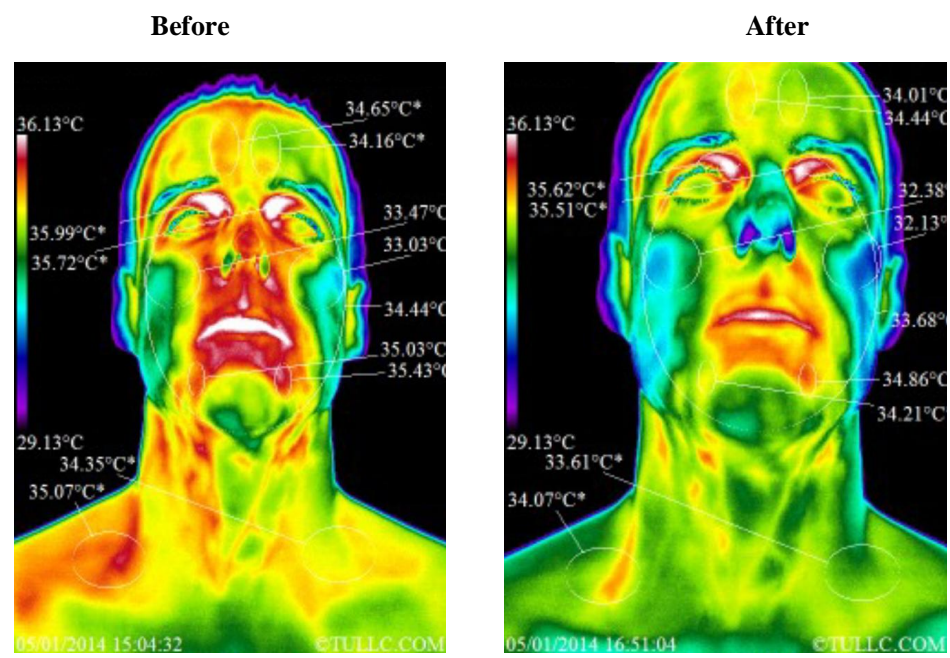

Figure 13. Subject D facial thermal images before and after a one hour shamgrounding session.
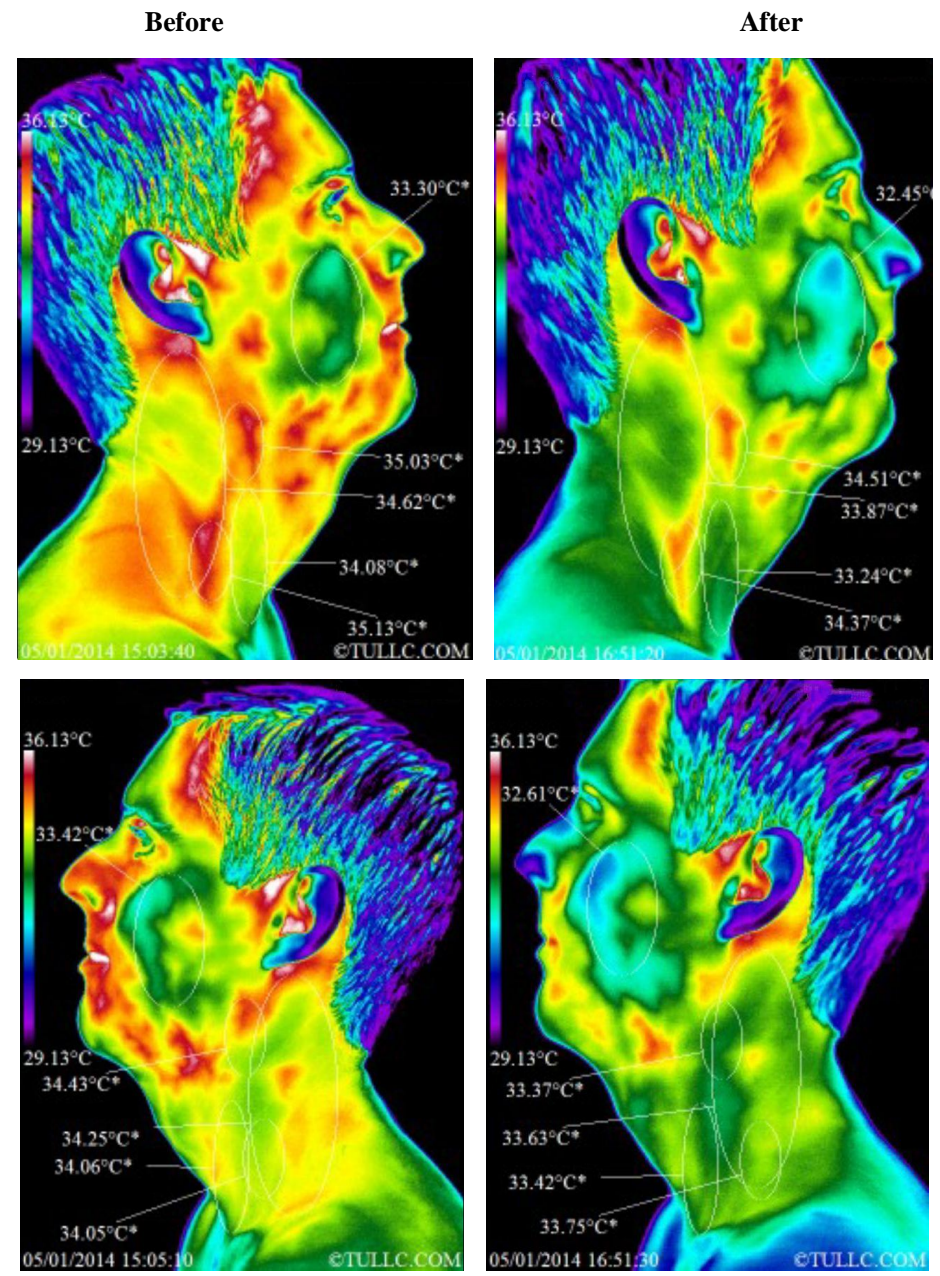

Figure 14. Top images: Subject D thermal images of the right lateral side of the face and neck before and after a one hour sham-grounding session. Bottom images: Subject $\mathrm{D}$ thermal images of the left lateral side of the face and neck before and after one hour of sham-grounding. 

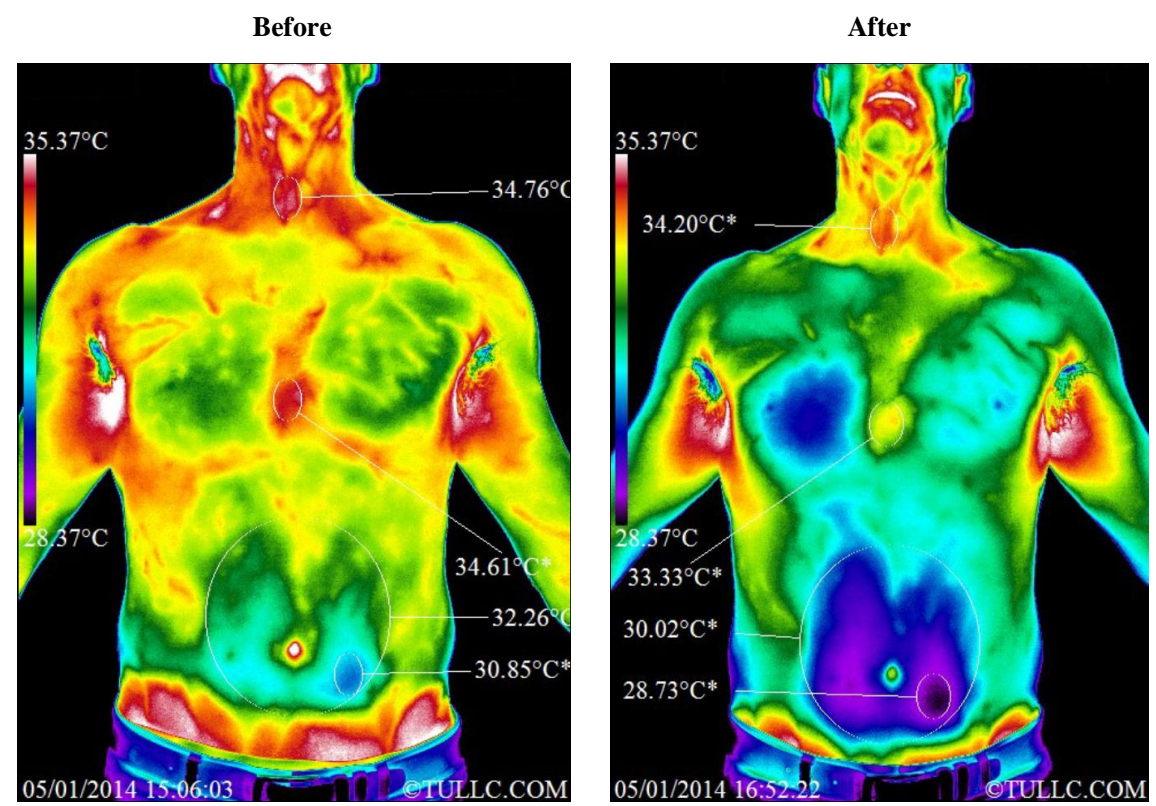

Figure 15. Subject D’s thermal images showing full anterior views of the torso before and after one hour of sham-grounding.

Before

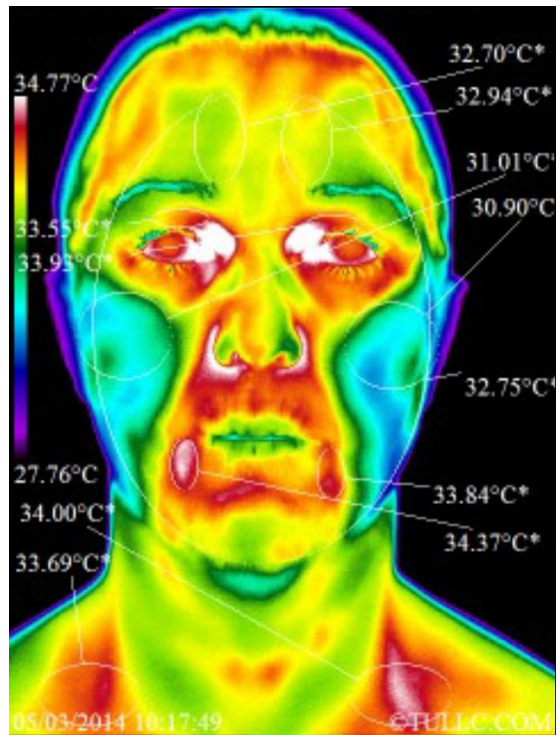

After

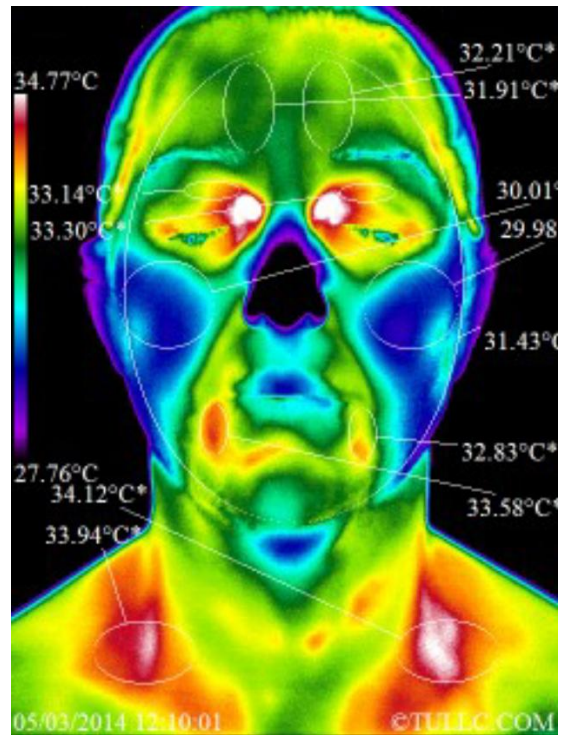

Figure 16. Subject E facial thermal images before and after a one hour sham-grounding session.

letter "P" superimposed on the regions of pain. The span and positioning of these images is between $27.76^{\circ} \mathrm{C}$ and $34.77^{\circ} \mathrm{C}$. The entire anterior torso cooled off except for the axilla and the supraclavicular regions. The temperature of the region around the umbilicus decreased significantly $\left(32.43^{\circ} \mathrm{C}-31.54^{\circ} \mathrm{C}=0.89^{\circ} \mathrm{C}\right)$. Statistical details are presented in Table 4.

3) Subject F-Female, African-American, 68, with left leg lateral pain and mild right lateral knee pain

Subject F's facial thermal images were analyzed using a span of $7^{\circ} \mathrm{C}$ and positioning between $28.22^{\circ} \mathrm{C}$ and $35.22^{\circ} \mathrm{C}$. Figure 19 shows that the temperature of the face decreased significantly after the sham-grounding session $\left(31.80^{\circ} \mathrm{C}-31.09^{\circ} \mathrm{C}=0.71^{\circ} \mathrm{C}\right)$. The temperature of the supraclavicular regions increased but not significantly and the temperature asymmetry between these regions, which was not significant to start with, decreased after 

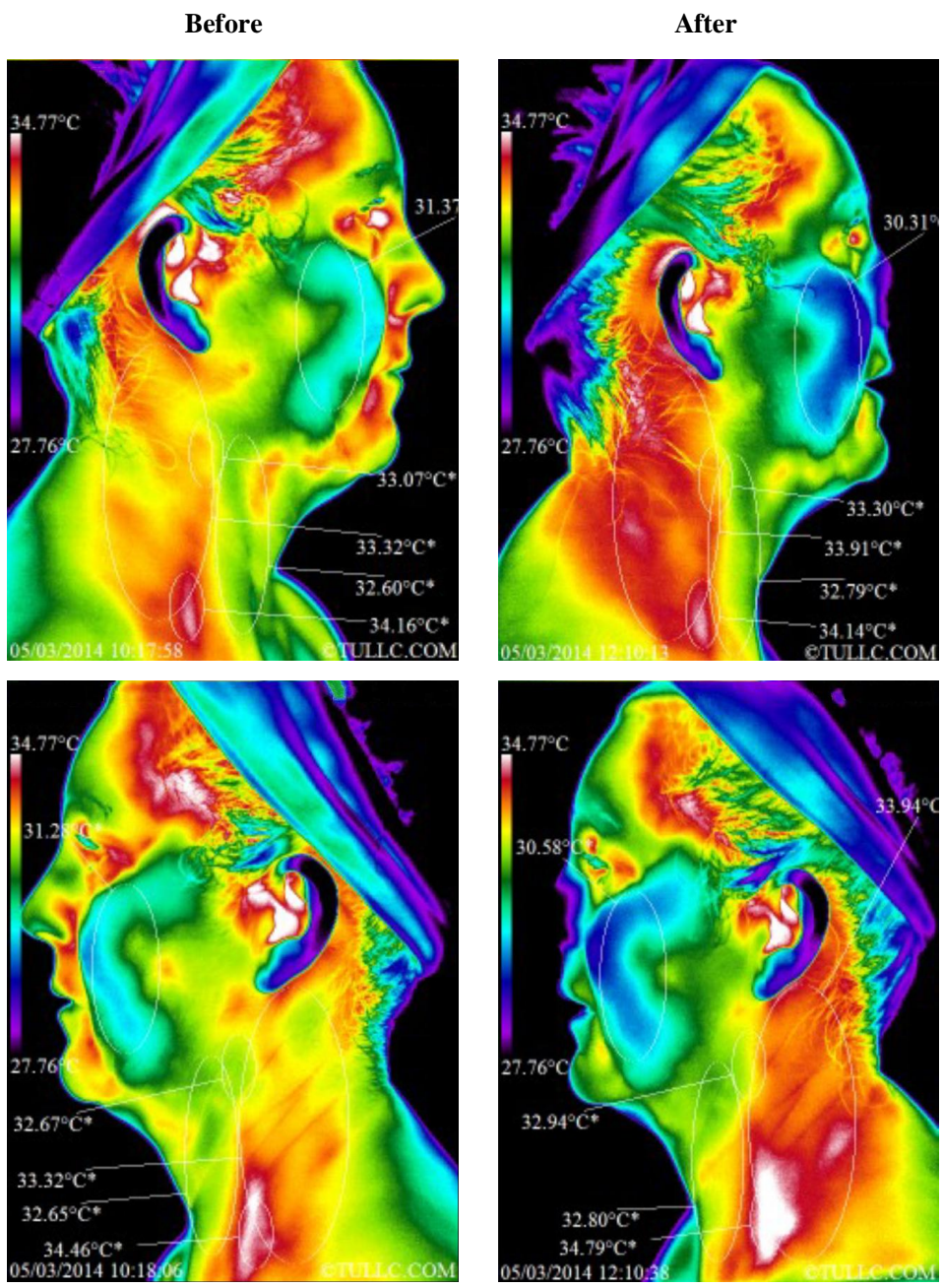

Figure 17. Top images: Subject $\mathrm{E}$ thermal images of the right lateral side of the face and neck before and after a one hour sham-grounding session. Bottom images: Subject $\mathrm{E}$ thermal images of the left lateral side of the face and neck before and after one hour of sham-grounding.
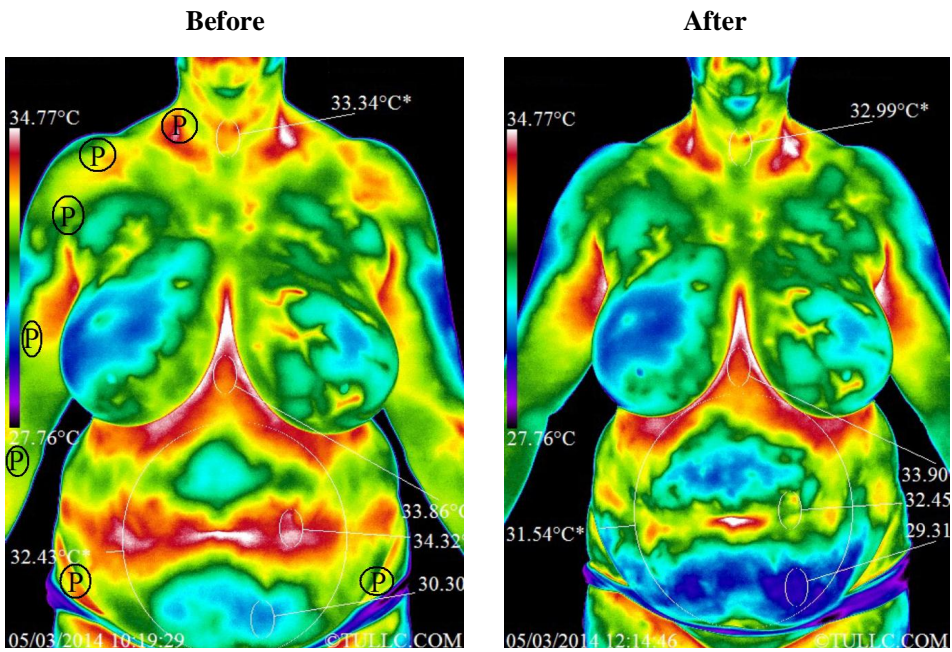

Figure 18. Subject E's thermal images showing full anterior views of the torso before and after one hour of sham-grounding. 

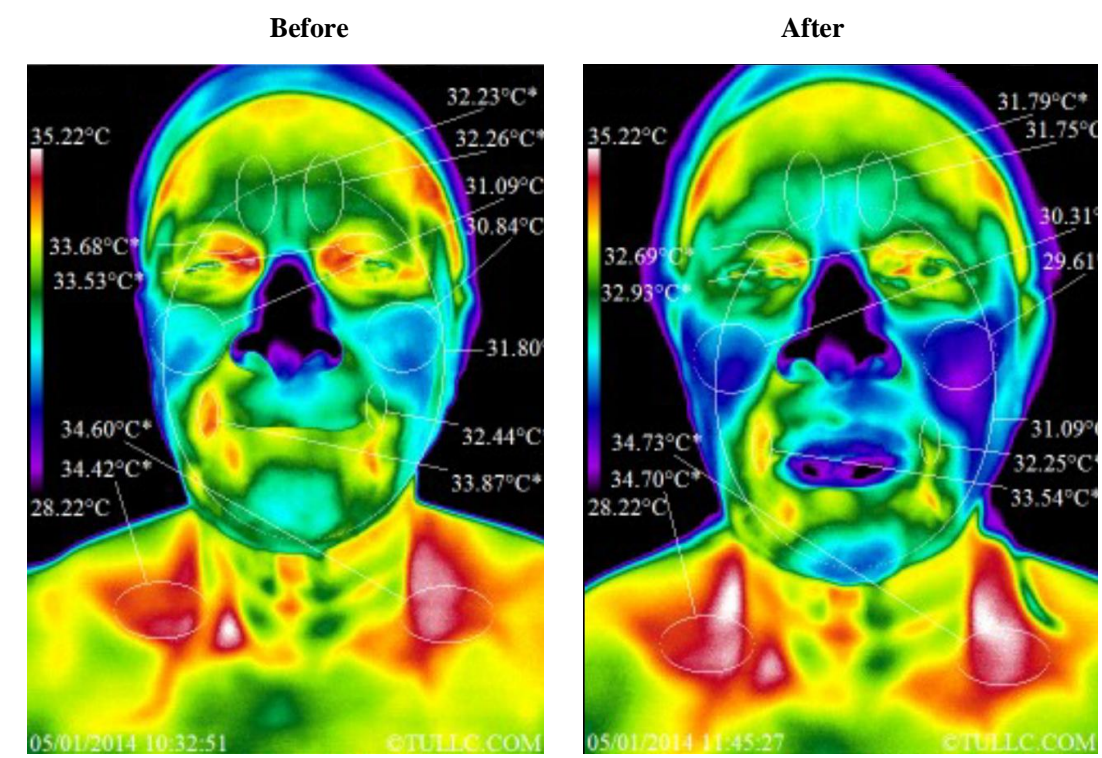

Figure 19. Subject $\mathrm{F}$ facial thermal images before and after one hour of sham-grounding.

the sham-grounding session. Statistical details of the image markers are presented in Table 1. Figure 20 top images show the right lateral side of the face and bottom images show the left lateral side of the face before and after a sham-grounding session. Here, thermal images reveal that the cheeks cooled off significantly (right side: $31.68^{\circ} \mathrm{C}-30.89^{\circ} \mathrm{C}=0.79^{\circ} \mathrm{C}$; left side: $31.63^{\circ} \mathrm{C}-30.41^{\circ} \mathrm{C}=1.22^{\circ} \mathrm{C}$ ) and that it is also the case for the right lateral aspects of the neck $\left(34.18^{\circ} \mathrm{C}-32.91^{\circ} \mathrm{C}=1.27^{\circ} \mathrm{C}\right)$. The left lateral aspects of the neck also cooled off but not significantly. The entire left lateral side of the face and neck cooled off except for the supraclavicular region which remained essentially at the same temperature. Statistical details are presented in Table 2. Figure 21 shows full anterior views of Subject F's torso before and after a sham-grounding session. The span and positioning is between $28.27^{\circ} \mathrm{C}$ and $35.27^{\circ} \mathrm{C}$. The entire anterior torso cooled off except around the anterior neck and the supraclavicular region on the right side. The temperature around the umbilicus did not decrease significantly. Statistical details are presented in Table 4.

\subsection{Clinical Observations}

\subsubsection{Grounded Subjects}

1) Subject A-Female, Caucasian, 33, with history of extensive pain

This subject has a history of multiple areas of pain indicated at a 3 on a scale from 1 - 10. Most of the entire neck/back/arms/forearms/thighs/legs dropped down to a 1 level after the grounding session. There are significant increases in temperature, from $1^{\circ} \mathrm{C}-3^{\circ} \mathrm{C}$, on almost all temperature markers, particularly over the abdomen. Low abdominal temperature markers had the most significant increases in temperature. For example, in Figure 6 the temperature of the marker on the left side above the umbilicus increased by a very significant $3.06^{\circ} \mathrm{C}$ $\left(33.93^{\circ} \mathrm{C}-30.87^{\circ} \mathrm{C}=3.06^{\circ} \mathrm{C}\right)$. The abdomen had a significant improvement in symmetry from hot to cold spot temperature difference of $4.02^{\circ} \mathrm{C}$ before the grounding session to $2.12^{\circ} \mathrm{C}$ after the grounding session, resulting in an overall better abdominal homogenous temperature pattern (Table 4 and Table 5). The chest radiating heat pattern toward both breasts (looking like a white $\mathrm{X}$ on the chest) is related to estrogen influences/lack of progesterone/improper clearing of hormones by the liver into bile.

All markers of Figure 4 increased in temperature after the grounding session. This is due to the entire torso surge of lymphatic/venous flow routing lymphatic fluids via the inferior vena cava/right subclavian vein/thoracic duct into the left subclavian vein. The upper torso fluids are also routed to the heart via the superior vena cava. This happened while grounded in order to produce a physiological homeostasis/stabilization of core temperature. This subject had what appears to be a purging of lymphatic/venous congestion that not all subjects experienced. As in Figure 4, all markers of Figure 5 increased in temperature. Significant overall head/neck increased temperatures appear to be related to cranial fluids competing with torso fluids that are being overly dumped into the same veins [14]-[17]. 

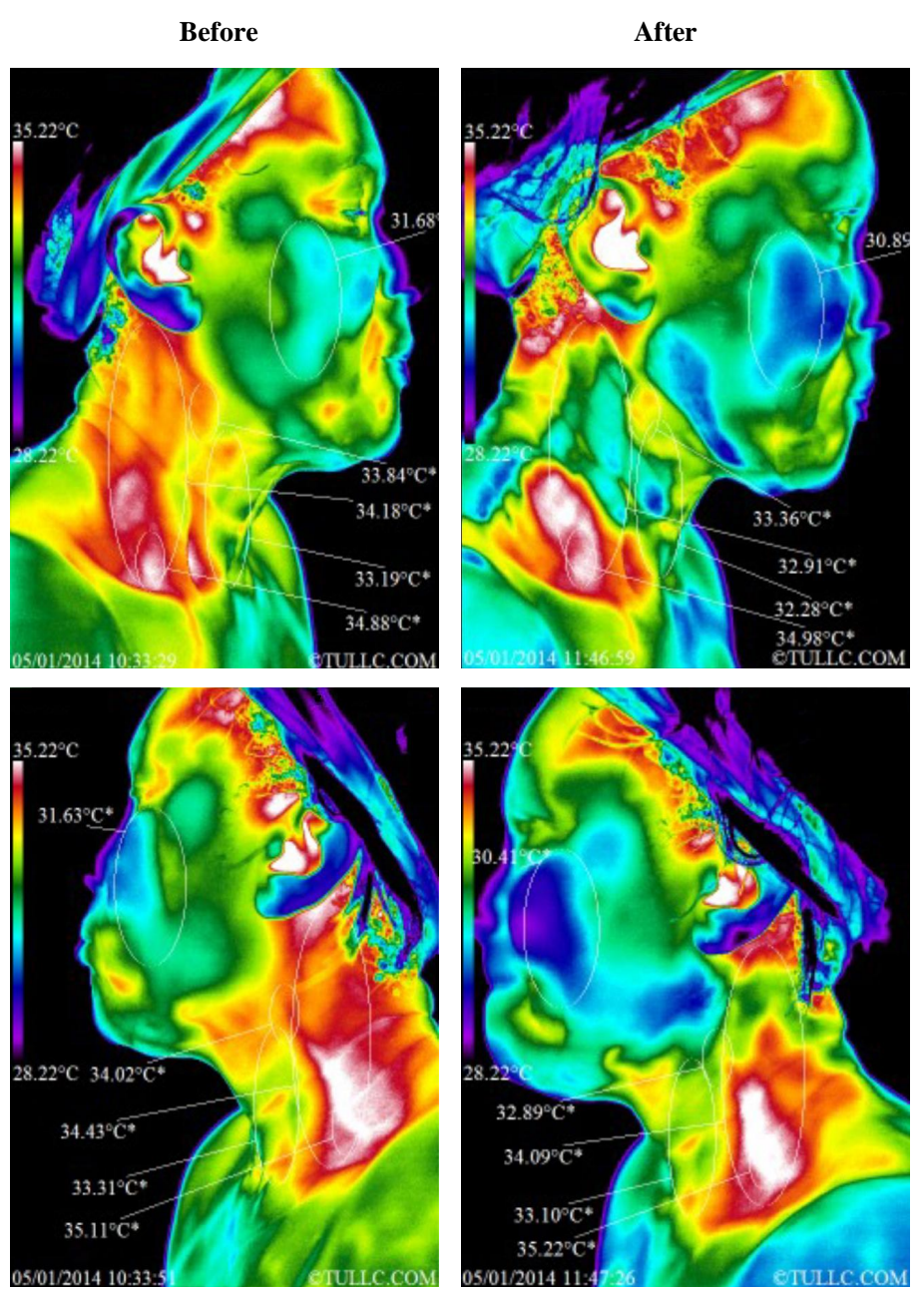

Figure 20. Top image: Subject $\mathrm{F}$ thermal images of the right lateral side of the face and neck before and after one hour of sham-grounding. Bottom image: Same subject thermal images of the left lateral side of the face and neck before and after one hour of sham-grounding.

Before

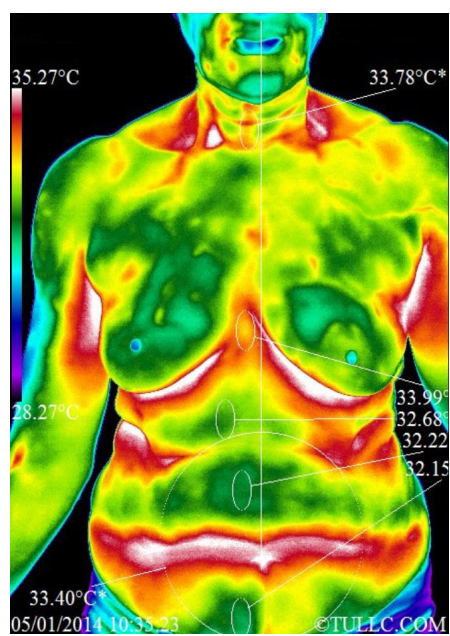

After

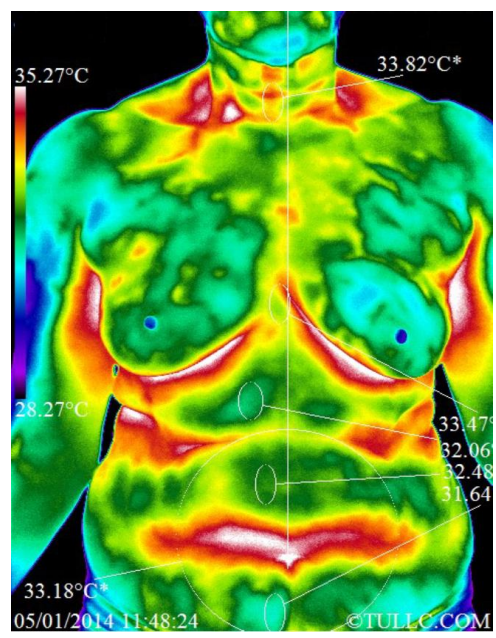

Figure 21. Subject F's thermal images showing full anterior views of the torso before and after one hour of sham-grounding. 
2) Subject B-Female, Caucasian, 49, with history of pain and surgery for right thumb and right lateral breast region

Subject B has a history of right thumb pain with surgery in the region and right lateral breast region pain of a mild intensity. Figure 7 shows significant drop in temperatures over the forehead/eye/oral regions. These indicate significant improved decongestion of the anterior region of the head/neck and significant drop in inflammatory response over the right lower periodontal disorder. There is significant improvement of the lymphatic drainage thermal marker along the right side of the throat to the sternum. Frequently, while viewing the supraclavicular region, one sees a temporary increase in temperature with the surge of lymphatic/venous fluids to the region, but in this case a significantly improved symmetry to the contralateral region and a drop in actual temperature can be seen. This type of drainage and symmetry indicates a healthier pattern overall. Figure 8 shows a significant drop in temperatures over the temporal and oral regions. These indicate improved temporalis myofascial activity with reduced temporomandibular joint compromising effects. There is also a significant reduction in the sternocliedo hypercontractive state. The external jugular vein is hot due to distension of the vein associated with bilateral scalenus myofascitis causing entrapment and back-pressure on this vein. Looking at Figure 9 the diaphragm/hiatal region of the stomach shows minimal changes, radiating lymphatic/venous congestion along the ascending/descending colon into the lower pelvic floor. There is a significant surge in lymphatic/ venous fluids from the lower extremities. The temperature of the colder areas of the abdomen (related to abdominal pressure) increased and this corresponds to a reduction in distention patterns. Overall temperatures improved from the diaphragm to the clavicle, with significant drop in anterior deltoid temperature and improved symmetry of the breasts.

3) Subject C-Female, Caucasian, 55, with history of uterine fibroids and lower pelvic pain

Subject $C$ has a history of 2 fibroid in the uterus with intermittent lower pelvic pain ranging from $2-4$, also right lower pelvic region. Figure 10 shows significant drop in temperatures over the following regions: forehead/eyes/orbit/surrounding nasal and oral regions. This is indicative of decongestion of the lymphatic/venous system of the head/neck with reduction in histamine type thermal markers surrounding the eyes and mouth. There is a significant reduction in periodontal inflammatory thermal markers that include the submandibular lymphatic system. The forehead heat pattern appears related to occiput/atlas entrapment of the suboccipital nerve/ vertebral artery resulting in dural influences affecting the 5th cranial nerve, thus causing frontalis/temporalis hypercontraction. In Figure 11, Subject C's temporalis and temporal mandibular joint had improvement in myofascial activity and reduction in joint compromising effects on both laterals. Yet, increased posterior neck temperatures appear as if this subject was influenced by the elevation of the pillow and headrest of the reclining chair. Significant drops in temperature over salivary gland sites, while the external jugular vein and the scalene region demonstrated consistent temperatures on the pre-post study. The images of the full anterior views of the torso shown in Figure 12 presented a drop in temperatures over the acromioclavicular joints, around the clavicles, much of the spotted breast fibrocystic activity, significant drop in lymphatic/venous congestion heat pattern surrounding both nipple and areola, specially on the right side, and temperature decreases in lymphatic/ venous congestion below the breast. However, the hiatal region over the stomach had only a small drop in temperature, while small intestine and lower pelvic thermal markers increased by more than $1^{\circ} \mathrm{C}$ with changes in abdominal distension pattern. Abdomen changes in pattern, like those observed around the umbilicus for this subject, are indicative of treatment/intervention effects (grounding). Autonomic control, or the lack there of, tends to produce consistent markers and patterns in the same geographical regions. Significant increased in vascular fluidity /digestive fluids/cerebral spinal fluid with improved autonomic control is related to intestinal lining motility. Clinical observations over the years have shown repeatedly that these changes in pattern do not happen without an external intervention.

\subsubsection{Sham-Grounded (Control) Subjects}

1) Subject D-Male, caucasian, 42, with bulging disc and left lateral foot numbness

Subject D had a bulging disc resulting in left lateral foot numbness. From Figure 13 and Figure 14 the forehead heat pattern appears related to pinched nerves or arteries between the back of head and neck thus affecting the nerves over the back of the head to the forehead. After one hour of sham-grounding, Subject D had a marked drop in eye/orbital/nasal/oral temperatures related to improved lymphatic/venous congestion. There is a reduction in histamine responses surrounding the eyes, nose and mouth with improved periodontal inflammation and 
reduction in lymphatic activity below the mandible with continued throat lymphatic activity. There also appears to be a bilateral maxillary/ethmoid sinus inflammation associated with improper cranial respiratory motion/lack of proper sinus aeration leading toward aggressive flora development. In Figure 14, saliva gland (parotid/submandibular/sublingual) heat patterns are suggestive of enlargement. The salivary glands may hypertrophy due to excessive stimulation by estrogen caused by iodine deficiency [18]. There is a mild to moderate temporomandibular joint (TMJ) inflammation with surrounding temporal muscular clenching that appears improved after the sham-grounding session. After 1 hour of sham-grounding, a significant drop in eye/orbital/nasal/oral inflammation with much improved lymph node below the jaw and throat inflammation can be seen. Figure 15 shows suspicious right upper chest heat pattern radiating toward right axilla/right supraclavicular regions associated with lymphatic/venous congestion. That heat pattern suggests diaphragm restricted movement/excessive abdominal pressure resulting in venous/lymphatic fluids to be congested at the diaphragm, then a re-routing of fluids along the chest/neck finally ending into the heart. The stomach heat pattern is suggestive of improper digestion/clearing of the stomach that can result in esophageal irritation/inflammation/hiatal pressure due to improper function of the pyloric valve. Small intestine distention can be seen associated with improper forwarding of stomach fluids/gallbladder fluids with issues related to an overly contracted spastic valve between the small and large intestines [14]-[17]. Small Intestine distention can apply excessive pressure into the lower pelvic floor resulting in lymphatic/venous congestion. After 1 hour of sham-grounding, an overall torso lymphatic/venous congestion improvement can be observed with the following results: $1.28^{\circ} \mathrm{C}\left(34.61^{\circ} \mathrm{C}-33.33^{\circ} \mathrm{C}=1.28^{\circ} \mathrm{C}\right)$ drop over the hiatal region of the stomach and $2^{\circ} \mathrm{C}$ drop of most of the entire abdomen (for example the region around the umbilicus was $32.26^{\circ} \mathrm{C}$ before and dropped to $30.02^{\circ} \mathrm{C}$ after the sham-grounded session, a decreased by $2.24^{\circ} \mathrm{C}$ ). However, the abdominal pattern of temperature did not change. For example, the cold spot on the lower left of the umbilicus before the session $\left(30.85^{\circ} \mathrm{C}\right)$ remained the coldest spot on the abdomen after the session $\left(28.73^{\circ} \mathrm{C}\right)$ while the hiatal region remained the warmest spot even while the entire torso cooled off. This is typical of a relaxation with no intervention situation.

2) Subject E-Female, caucasian, 55, with bilateral pelvic pain and inner right arm pain from shoulder to hand

According to Figure 16, there is a significant drop in temperatures surrounding the orbital/nasal/oral and frontal/temporal regions related to decongestion of lymphatic/venous systems from the head/neck. The face had an average decrease in temperatures of $1.32^{\circ} \mathrm{C}\left(32.75^{\circ} \mathrm{C}-31.43^{\circ} \mathrm{C}=1.32^{\circ} \mathrm{C}\right)$ while the left and right supraclavicular regions had a slight increase by $0.12^{\circ} \mathrm{C}$ and $0.25^{\circ} \mathrm{C}$, respectively, suggestive of increases in lymphatic/ venous fluids routed through the subclavian vein. Figure 17 lateral neck image marker evaluations show increased temperatures indicative of a surge of lymphatic/venous fluids routed into the subclavian vein. Posterior-lateral cervicocranial temperatures also appear to be related to myofascial activity that can be influenced by the head elevation with pillow and reclining chair headrest. From Figure 18 after the sham-grounding session, there is an overall abdominal decrease in temperatures except for the stomach/hiatal region. These results appear to be due to improved lymphatic/venous congestion. Diaphragmatic breathing influences unidirectional valves of both lymphatic and venous flow. After the cistern of chyli, a dilated region of the lymphatic system below the diaphragm and prior to the thoracic duct, the diaphragm wall has a significant influence in the forward movement of lymph through the thoracic duct into the left subclavian vein. A significant drop in abdominal temperature of $0.89^{\circ} \mathrm{C}\left(32.43^{\circ} \mathrm{C}-31.54^{\circ} \mathrm{C}=0.89^{\circ} \mathrm{C}\right)$ is suggestive of an improved venous/lymph return that appeared to be due to the subject relaxing in a comfortable reclining chair which made the existing distention more apparent with no indication of an intervention (grounding).

3) Subject F-Female, African-American, 68, with left leg lateral pain and mild right lateral knee pain

This subject indicated that she had pain along the left lateral thigh/leg with mild right lateral knee pain. Figure 19 shows significant or almost significant temperature drops in forehead/eye/orbital/cheeks/periodontal sites. The face had an average decrease in temperature of $0.71^{\circ} \mathrm{C}\left(31.80^{\circ} \mathrm{C}-31.09^{\circ} \mathrm{C}=0.71^{\circ} \mathrm{C}\right)$ while the left and right supraclavicular regions had insignificant temperature increases by $0.13^{\circ} \mathrm{C}$ and $0.28^{\circ} \mathrm{C}$, respectively, suggestive of an increased lymphatic/venous fluids routed through the subclavian vein. From Figure 20, significant drops in cheeks/periodontal sites/left TMJ/upper lateral-posterior neck temperature and in lymphatic/venous congestion can be seen. Slight increases in temperature are noted over the following regions: right occiput/scalenus/ supraclavicular region/right lower-anterior lymphatic involvement (localized)/right TMJ. Figure 21 shows a significant drop of temperatures in the hiatal region of the stomach $\left(33.99^{\circ} \mathrm{C}-33.47^{\circ} \mathrm{C}=0.52^{\circ} \mathrm{C}\right)$. In general 
abdominal sites had decreased temperatures. These effects can be attributed to relaxation with improved lymphatic/venous congestion without intervention (grounding). Decreases in abdominal temperatures are related to an increased abdominal distention, suggestive of an improved venous/lymph return that appeared to be due to the subject relaxing in a comfortable reclining chair which made the existing distention more apparent.

\subsection{Statistical Analyses}

\subsubsection{Face Anterior View}

Table 1 shows before vs. after temperature marker values from the face anterior view thermal images for within and between group comparisons. Temperature differences above $0.5^{\circ} \mathrm{C}$ are considered significant as per the discussion in Section 2.4 Statistical Analysis. From Table 1, it can be seen that each subject had a statistically significant change in average temperature marker after the grounded session or the sham-grounded (see columns with heading “Diff”). Looking at the last 3 columns entitled “Grounded Group Avg.” and “Control Group Avg.”, which present the average temperature of the markers of the 3 subjects in their respective group, the grounded group shows a decrease in average temperature of less than $0.5^{\circ} \mathrm{C}\left(0.25^{\circ} \mathrm{C}\right)$ after grounding that is barely statistically significant ( $p=0.0423$ ) while the sham-grounded (control) group presents a decrease in temperature above $0.5^{\circ} \mathrm{C}\left(0.64^{\circ} \mathrm{C}\right)$ that is highly statistically significant $(p=0.00008)$. Between group comparisons show that the average temperature of the face for both groups before the session was similar $(p=0.564)$ and this was also the case after the session $(p=0.958)$. In other words, the statistical tests could not rule out that the two groups had the same temperature before and after the session. However, the average temperature difference (last column) is significantly smaller for the grounded group ( $p=0.002)$, meaning that the smaller decrease in temperature observed for the grounded group compared to the control group is not a chance happening but that grounding must have played a significant role in producing that difference in average temperature between groups. Left to right differences before and after grounding and between groups were also calculated but did not show any significant result for the face anterior view thermal images.

\subsubsection{Face Lateral Views}

Table 2 shows statistical results comparing before vs. after session marker temperature values within and between groups for the left and right lateral views of the face. Again, temperature differences above $0.5^{\circ} \mathrm{C}$ are considered significant. Two subjects in each group show a statistically significant change in temperature comparing before and after the session (subjects A, B, D, and F). The results for the average of the three subjects of each group are shown in the last three columns entitled “Grounded Group Avg.” and “Control Group Avg.”, respectively. There is an insignificant decrease in average temperature for the grounded group of $0.04^{\circ} \mathrm{C}(p=0.878)$ while the sham-grounded group shows a decrease in average temperature by $0.42^{\circ} \mathrm{C}$, a statistically significant decrease $(p=0.00256)$. The average temperatures between groups before the session and after the session are similar ( $p=0.213$ and $p=0.791$, respectively). The statistical tests could not rule out that the two groups had the same temperature before the session and also after the session. However, similarly to the results of the face anterior view images, the average temperature difference was statistically different with the grounded group having a significantly smaller average temperature decrease than the control group $(p=0.017)$. The similarity of the results presented in Table 1 and Table 2 is to be expected since we are looking at the same faces but from different angles.

Table 3 presents the same results as Table 2 but reorganized to emphasized left-right symmetry. In this table, the absolute difference between left and right lateral views (labeled “Abs Diff”, taking the absolute value makes the difference always positive) was calculated for each subject and each temperature marker before and after the session. The difference between left-right absolute differences was then calculated by subtracting the "Before" absolute difference from the "After" absolute difference. The result of this calculation is presented in the last column labeled “ $\Delta$ Diff” i.e. $\Delta$ Diff $=$ After Abs Diff - Before Abs Diff. If $\Delta$ Diff is positive, the implications are that there was an increase in temperature difference between left and right lateral views of the face after the session for that particular temperature marker and subject i.e., the symmetry as decreased (the more positive $\Delta$ Diff is the larger the symmetry decrease). The opposite is true if $\Delta \mathrm{Diff}$ is negative. As can be seen, $\Delta \mathrm{Diff}$ decreased by $0.10^{\circ} \mathrm{C}$ on average for the grounded group while it increased by $0.19^{\circ} \mathrm{C}$ on average for the sham-grounded group. This difference in $\Delta$ Diff is statistically significant $(p=0.0284)$ meaning that the left-right facial symmetry improvement for the grounded group as compared to the control group must be due to a real factor (grounding). 
Table 3. Face right and left lateral views symmetry statistics.

\begin{tabular}{|c|c|c|c|c|c|c|c|}
\hline \multirow{3}{*}{$\begin{array}{l}\text { Subjects: } \\
\text { Marker }\end{array}$} & \multicolumn{7}{|c|}{ Grounded Group } \\
\hline & \multicolumn{2}{|c|}{ Before } & \multicolumn{3}{|c|}{ After } & \multirow[b]{2}{*}{ Abs Diff } & \multirow[b]{2}{*}{$\Delta$ Diff } \\
\hline & $\mathbf{L}$ & $\mathbf{R}$ & Abs Diff & $\mathbf{L}$ & $\mathbf{R}$ & & \\
\hline \multirow{3}{*}{ Cheeks } & 30.44 & 30.55 & 0.11 & 30.72 & 30.92 & 0.20 & 0.09 \\
\hline & 31.28 & 31.91 & 0.63 & 30.57 & 30.34 & 0.23 & -0.40 \\
\hline & 31.64 & 31.14 & 0.50 & 30.81 & 30.12 & 0.69 & 0.19 \\
\hline \multirow[t]{3}{*}{ Carotid Bif. } & 32.91 & 32.14 & 0.77 & 33.12 & 33.12 & 0.00 & -0.77 \\
\hline & 33.89 & 34.08 & 0.19 & 33.65 & 33.97 & 0.32 & 0.13 \\
\hline & 33.61 & 33.50 & 0.11 & 33.21 & 33.25 & 0.04 & -0.07 \\
\hline \multirow[t]{3}{*}{ Back Neck } & 33.73 & 33.10 & 0.63 & 33.95 & 33.92 & 0.03 & -0.60 \\
\hline & 33.94 & 34.00 & 0.06 & 33.76 & 33.93 & 0.17 & 0.11 \\
\hline & 33.79 & 33.67 & 0.12 & 33.75 & 34.15 & 0.40 & 0.28 \\
\hline \multirow[t]{3}{*}{ Front Neck } & 32.26 & 31.71 & 0.55 & 32.73 & 32.82 & 0.09 & -0.46 \\
\hline & 33.36 & 33.27 & 0.09 & 33.01 & 32.56 & 0.45 & 0.36 \\
\hline & 32.51 & 32.54 & 0.03 & 32.59 & 32.60 & 0.01 & -0.02 \\
\hline \multirow[t]{3}{*}{ Supraclav. } & 34.64 & 34.20 & 0.44 & 34.76 & 34.93 & 0.17 & -0.27 \\
\hline & 34.61 & 34.24 & 0.37 & 34.22 & 33.99 & 0.23 & -0.14 \\
\hline & 33.61 & 33.66 & 0.05 & 33.80 & 33.61 & 0.19 & 0.14 \\
\hline Average: & 33.08 & 32.91 & -0.17 & 32.98 & 32.95 & -0.03 & -0.10 \\
\hline SD: & 1.23 & 1.18 & -0.05 & 1.31 & 1.44 & 0.14 & 0.34 \\
\hline Normality & & & & & & & Yes \\
\hline \multirow{2}{*}{ Subjects: } & \multicolumn{3}{|c|}{ Control Group } & & & & \\
\hline & \multicolumn{2}{|c|}{ Before } & \multicolumn{3}{|c|}{ After } & & \\
\hline Marker & $\mathbf{L}$ & $\mathbf{R}$ & Abs Diff & $\mathbf{L}$ & $\mathbf{R}$ & Abs Diff & $\Delta$ Diff \\
\hline \multirow[t]{3}{*}{ Cheeks } & 33.42 & 33.30 & 0.12 & 32.61 & 32.45 & 0.16 & 0.04 \\
\hline & 31.28 & 31.37 & 0.09 & 30.58 & 30.31 & 0.27 & 0.18 \\
\hline & 31.63 & 31.68 & 0.05 & 30.41 & 30.89 & 0.48 & 0.43 \\
\hline \multirow[t]{3}{*}{ Carotid Bif. } & 34.33 & 35.03 & 0.70 & 33.37 & 34.51 & 1.14 & 0.44 \\
\hline & 32.67 & 33.07 & 0.40 & 32.94 & 33.30 & 0.36 & -0.04 \\
\hline & 34.02 & 33.84 & 0.18 & 32.89 & 33.36 & 0.47 & 0.29 \\
\hline \multirow[t]{3}{*}{ Back Neck } & 34.25 & 34.62 & 0.37 & 33.63 & 33.87 & 0.24 & -0.13 \\
\hline & 33.32 & 33.32 & 0.00 & 33.94 & 33.91 & 0.03 & 0.03 \\
\hline & 34.43 & 34.18 & 0.25 & 34.09 & 32.91 & 1.18 & 0.93 \\
\hline \multirow[t]{3}{*}{ Front Neck } & 34.06 & 34.08 & 0.02 & 33.42 & 33.24 & 0.18 & 0.16 \\
\hline & 32.65 & 32.60 & 0.05 & 32.80 & 32.79 & 0.01 & -0.04 \\
\hline & 33.31 & 33.19 & 0.12 & 33.10 & 32.28 & 0.82 & 0.70 \\
\hline \multirow[t]{3}{*}{ Supraclav. } & 34.05 & 35.13 & 1.08 & 33.75 & 34.37 & 0.62 & -0.46 \\
\hline & 34.46 & 34.16 & 0.30 & 34.79 & 34.14 & 0.65 & 0.35 \\
\hline & 35.11 & 34.88 & 0.23 & 35.22 & 34.98 & 0.24 & 0.01 \\
\hline Average: & 33.53 & 33.63 & 0.10 & 33.17 & 33.15 & -0.02 & 0.19 \\
\hline SD: & 1.08 & 1.14 & 0.06 & 1.30 & 1.29 & -0.01 & 0.35 \\
\hline Normality & & & & & & & Yes \\
\hline Equal variance: & & & & & & & Yes \\
\hline t-test: & & & & & & & 0.0284 \\
\hline
\end{tabular}

L = Left; $\mathrm{R}=$ Right; Supraclav. = Supraclavicular; Bif. = Bifurcation. 


\subsubsection{Full Anterior Torso Views}

Table 4 shows the results of the before vs. after marker temperature differences for full anterior torso view comparisons between groups. The last three columns entitled "Grounded Group Avg" or "Control Group Avg." show that the average temperature between groups before and after the session were similar ("Before" $p=0.626$; "After" $p=0.168)$. However, the average temperature differences were statistically significant with the grounded group having an average temperature increase by a significant $0.88^{\circ} \mathrm{C}$ while the control sham-grounded group had a significant temperature decrease of $1.20^{\circ} \mathrm{C}(p=0.00212)$, indicative of relaxation.

Also presented in Table 4 is the difference between the maximal (hot) and minimal (cold) abdominal temperature of 4 temperature markers ("Around Umbilicus"; "Btwn Breasts"; Abd Cold Spot”; "Abd Hot Spot”) before and after the session ("Before" columns and "After" columns) for each subject and the group average before and after the session as well as their difference ("Diff" columns). This is presented in the first row of numbers

\section{Table 4. Full anterior torso view statistics.}

\begin{tabular}{|c|c|c|c|c|c|c|c|c|c|c|c|c|}
\hline \multirow{2}{*}{\begin{tabular}{|l} 
Subject: \\
Marker
\end{tabular}} & \multicolumn{3}{|c|}{ A } & \multicolumn{3}{|c|}{ B } & \multicolumn{3}{|c|}{ C } & \multicolumn{3}{|c|}{ Grounded Group Avg. } \\
\hline & Before & After & Diff & Before & After & Diff & Before & After & Diff & Before & After & Diff \\
\hline Abd Hot-Cold: & 4.02 & 2.12 & -1.90 & 2.31 & 1.37 & -0.94 & 4.60 & 3.20 & -1.40 & 3.64 & 2.23 & -1.41 \\
\hline Around Umbilicus & 31.84 & 34.35 & 2.51 & 32.24 & 32.74 & 0.50 & 32.06 & 32.29 & 0.23 & 32.05 & 33.13 & 1.08 \\
\hline Btwn Breasts & 34.52 & 34.96 & 0.44 & 33.43 & 33.57 & 0.14 & 33.64 & 33.43 & -0.21 & 33.86 & 33.99 & 0.12 \\
\hline Abd Cold Spot & 30.50 & 33.09 & 2.59 & 31.42 & 32.39 & 0.97 & 29.04 & 30.23 & 1.19 & 30.32 & 31.90 & 1.58 \\
\hline Abd Hot Spot & 33.74 & 35.21 & 1.47 & 33.73 & 33.76 & 0.03 & & & & 33.74 & 34.49 & 0.75 \\
\hline Average: & 32.65 & 34.40 & 1.75 & 32.71 & 33.12 & 0.41 & 31.58 & 31.98 & 0.40 & 32.49 & 33.38 & 0.88 \\
\hline SD: & 1.82 & 0.95 & 0.88 & 1.07 & 0.66 & -0.42 & 2.34 & 1.62 & 0.72 & 1.67 & 1.13 & 0.54 \\
\hline t-test: & \multicolumn{2}{|c|}{0.0406} & \multicolumn{4}{|c|}{0.149} & \multicolumn{2}{|c|}{0.432} & \multicolumn{4}{|c|}{0.0631} \\
\hline Normality: & Yes & Yes & Yes & Yes & Yes & Yes & Yes & Yes & Yes & Yes & Yes & Yes \\
\hline Subject: & \multicolumn{3}{|c|}{ D } & \multicolumn{3}{|c|}{$\mathbf{E}$} & \multicolumn{3}{|c|}{$\mathbf{F}$} & \multicolumn{3}{|c|}{ Control Group Avg. } \\
\hline Marker & Before & After & Diff & Before & After & Diff & Before & After & Diff & Before & After & Diff \\
\hline Abd Hot-Cold: & 3.76 & 4.60 & 0.84 & 4.02 & 4.59 & 0.57 & 1.84 & 1.83 & -0.01 & 3.21 & 3.67 & 0.47 \\
\hline Around Umbilicus & 32.26 & 30.02 & -2.24 & 32.43 & 31.54 & -0.89 & 33.40 & 33.18 & -0.22 & 32.70 & 31.58 & -1.12 \\
\hline Btwn Breasts & 34.61 & 33.33 & -1.28 & 33.86 & 33.90 & 0.04 & 33.99 & 33.47 & -0.52 & 34.15 & 33.57 & -0.59 \\
\hline Abd Cold Spot & 30.85 & 28.73 & -2.12 & 30.30 & 29.31 & -0.99 & 32.15 & 31.64 & -0.51 & 31.10 & 29.89 & -1.21 \\
\hline Abd Hot Spot & & & & 34.32 & 32.45 & -1.87 & & & & 34.32 & 32.45 & -1.87 \\
\hline Average: & 32.57 & 30.69 & -1.88 & 32.73 & 31.80 & -0.93 & 33.18 & 32.76 & -0.42 & 33.07 & 31.87 & -1.20 \\
\hline SD: & 1.90 & 2.37 & 0.47 & 1.81 & 1.92 & 0.12 & 0.94 & 0.98 & 0.04 & 1.50 & 1.55 & 0.05 \\
\hline t-test: & \multicolumn{2}{|c|}{0.0248} & \multicolumn{4}{|c|}{0.0980} & \multicolumn{2}{|c|}{0.0515} & \multicolumn{4}{|c|}{0.0200} \\
\hline Normality: & Yes & Yes & Yes & Yes & Yes & Yes & Yes & Yes & Yes & Yes & Yes & Yes \\
\hline t-test: & & & & & & & & & & 0.626 & 0.168 & 0.002 \\
\hline Normality: & & & & & & & & & & Yes & Yes & Yes \\
\hline Equal Variance: & & & & & & & & & & Yes & Yes & Yes \\
\hline
\end{tabular}

Abd = Abdominal; Btwn = Between . 
(entitled on the left "Abd Hot-Cold"), which are calculated by looking at the highest temperature and lowest temperature of the 4 markers. For example, looking at subject A before the session (column entitled "Before"), the abdominal hot-cold maximum difference is $4.02^{\circ} \mathrm{C}$ and it is the difference between $34.52^{\circ} \mathrm{C}$ (between the breasts) and $30.50^{\circ} \mathrm{C}$ (abdominal cold spot). The same is done for after the session and for the other 5 subjects. The average after-minus-before session difference in max-min temperatures (columns entitled "Diff") decreased by a significant $1.41^{\circ} \mathrm{C}$ for the grounded group while it increased by $0.47^{\circ} \mathrm{C}$, a temperature difference of $1.88^{\circ} \mathrm{C}$ between groups. This means that the temperature of the abdomen became more homogeneous for the grounded subjects (less difference between the hot and cold markers after the grounded session) while it became more inhomogeneous for the sham-grounded subjects (increased difference between hot and cold markers after the sham-grounded session).

Table 5 presents the abdominal hot and cold spot statistics of Table 4 in more details. Even though there are only 3 subjects in each group, there is enough difference between groups' hot/cold marker differences to permit a statistical determination that the grounded group had a significantly smaller hot/cold temperature difference as compared to the sham-grounded (control) group $(p=0.0073)$.

\subsubsection{Combined}

To test the first hypothesis, the results of the face and torso have been combined and the average and standard deviation presented as well as statistical test results in Table 6. This table shows that, even though each subject of the grounded group had a significant before/after temperature difference, the grounded group average did not show a significant change $(p=0.948)$. This is because subject A had a significant increase while subjects B and $\mathrm{C}$ had significant decreases in their temperature markers. Contrastingly, each subject of the control shamgrounded group had a significant decrease in marker's temperature resulting in a significant control group average temperature decrease of $0.64^{\circ} \mathrm{C}(p=0.00005)$. The between group temperature differences were statistically significant $(p=0.00001)$. Even though 2 grounded subjects had temperature decreases, by $0.36^{\circ} \mathrm{C}$ and

Table 5. Torso hot minus cold temperature marker comparison statistics.

\begin{tabular}{|c|c|c|c|}
\hline Subject & Before & After & Diff \\
\hline A & 4.02 & 2.12 & -1.90 \\
\hline B & 2.31 & 1.37 & -0.94 \\
\hline C & 4.60 & 3.20 & -1.40 \\
\hline Average: & 3.64 & 2.23 & -1.41 \\
\hline SD: & 1.19 & 0.92 & 0.48 \\
\hline t-test: & \multicolumn{2}{|c|}{0.0364} & \\
\hline Normality: & Yes & Yes & Yes \\
\hline Subject & Before & After & Diff \\
\hline D & 3.76 & 4.60 & 0.84 \\
\hline $\mathbf{E}$ & 4.02 & 4.59 & 0.57 \\
\hline $\mathbf{F}$ & 1.84 & 1.83 & -0.01 \\
\hline Average: & 3.21 & 3.67 & 0.47 \\
\hline SD: & 1.19 & 1.60 & 0.43 \\
\hline Normality: & Yes & No & Yes \\
\hline W S-R test: & & & \\
\hline t-test: & 0.677 & 0.246 & 0.0073 \\
\hline Normality: & Yes & Yes & Yes \\
\hline Equal Variance: & Yes & Yes & Yes \\
\hline
\end{tabular}


Table 6. Combined face and torso statistics.

\begin{tabular}{|c|c|c|c|c|c|c|c|c|c|c|c|c|}
\hline \multirow{2}{*}{ Subject: } & \multicolumn{3}{|c|}{ A } & \multicolumn{3}{|c|}{ B } & \multicolumn{3}{|c|}{ C } & \multicolumn{3}{|c|}{ Grounded Group Avg. } \\
\hline & Before & After & Diff & Before & After & Diff & Before & After & Diff & Before & After & Diff \\
\hline Average: & 32.59 & 33.34 & 0.75 & 33.38 & 33.02 & -0.36 & 32.74 & 32.46 & -0.28 & 32.91 & 32.97 & 0.06 \\
\hline SD: & 1.50 & 1.44 & -0.07 & 1.32 & 1.34 & 0.02 & 1.27 & 1.34 & 0.07 & 1.29 & 1.32 & 0.03 \\
\hline Normality: & Yes & No & No & No & No & Yes & No & No & Yes & No & No & No \\
\hline W S-R test: & & & 0.00004 & & & 0.00386 & & & 0.0285 & & & 0.948 \\
\hline \multirow{2}{*}{ Subject: } & \multicolumn{3}{|c|}{ D } & \multicolumn{3}{|c|}{$\mathbf{E}$} & \multicolumn{3}{|c|}{$\mathbf{F}$} & \multicolumn{3}{|c|}{ Control Group Avg. } \\
\hline & Before & After & Diff & Before & After & Diff & Before & After & Diff & Before & After & Diff \\
\hline Average: & 34.17 & 33.36 & -0.81 & 32.87 & 32.44 & -0.43 & 33.13 & 32.55 & -0.58 & 33.41 & 32.77 & -0.64 \\
\hline SD: & 1.19 & 1.64 & 0.45 & 1.23 & 1.54 & 0.31 & 1.22 & 1.45 & 0.23 & 1.07 & 1.32 & 0.25 \\
\hline t-test: & & & & & & 0.00624 & & & 0.00001 & & & \\
\hline Normality: & No & No & No & Yes & Yes & Yes & Yes & Yes & Yes & No & Yes & No \\
\hline W S-R test: & & & 0.00006 & & & & & & & & & 0.00005 \\
\hline t-test: & & & & & & & & & & 0.174 & 0.615 & \\
\hline Normality: & & & & & & & & & & Yes & Yes & No \\
\hline Equal Variance: & & & & & & & & & & Yes & Yes & Yes \\
\hline W R-S test: & & & & & & & & & & & & 0.00001 \\
\hline
\end{tabular}

$0.28^{\circ} \mathrm{C}$ respectively, these decreases were less than even the smallest decrease of the control group which was $0.43^{\circ} \mathrm{C}$ (for subject E).

\section{Discussion}

The appearance of facial skin is a key indicator of perceived age and aging. The purpose of this study was to determine if grounding the body promotes blood flow to the face, which, if confirmed, would indicate an increase of blood-borne nutrients to the microcirculation of the skin and a subsequent improvement in facial vitality and appearance. For this purpose both LSCI and TI were used. These two methods of measurement have been shown to correlate well [19]. In a previous paper on the same study, LSCI was able to show that grounding improved facial blood flow regulation by making facial blood flow fluctuate with a regular rhythm and/or increase during the grounding session while no such fluctuation or increase was seen for sham-grounded control subjects [7]. The low frequency of the periodicity of facial blood flow changes was found to be in the frequency range of some of the baroreflex frequencies [7] [20] [21]. To understand how grounding could improve facial blood flow regulation, it is logical to also consider blood and lymphatic fluid circulation in the torso since blood flows from the torso through the neck to reach the head and the face. This is what we endeavored to do with the TI scans. Clinicians who regularly use thermal imaging find that TI can assist with understanding lymphatic fluid flow and functional metabolism in their patients [14]-[17].

Hypothesis 1 (H1) stated that there will be a statistically significant increase in skin temperature as measured by the TI camera when comparing images of specific skin regions of the face, neck, and torso before grounding and after the grounding period (no change is expected for the sham-grounding period). Looking at Table 1, which presents face frontal view results, a statistically significant decrease in average temperature of the markers can be seen for the grounded group $(p=0.0423)$ while a much more statistically significant decrease can be seen for the control group $(p=0.00008)$. The difference in temperature differences between groups after the session is statistically significant $(p=0.002)$, meaning the decrease in average temperature of the control group was significantly more pronounced than that of the grounded group. The results presented in Table 2 for the lateral views of the face are very similar. There was almost no change in average temperature after the session for 
the grounded group ( $p=0.878$ ) while there was a significant decrease in average temperature for the control group $(p=0.00256)$. The temperature difference between groups was also significant $(p=0.017)$. The results for the abdomen presented in Table 4 show an increase in temperature greater than $0.5^{\circ} \mathrm{C}$ for the grounded group $\left(0.88^{\circ} \mathrm{C}\right.$, although not statistically significant) and a statistically significant decrease in temperature for the control group $\left(1.20^{\circ} \mathrm{C} ; p=0.0200\right)$. From these results it is concluded that $\mathrm{H} 1$ was not supported, except for an increase in temperature of the abdomen for the grounded group. However, the two groups presented very different results. For the grounded group, average temperature changes in the face were not significant while a significant increase in average abdominal temperature was observed. For the control group, statistically significant decreases in average temperatures for both the face and abdominal region were found and these were significantly lower than the corresponding average temperatures for the grounded group.

Hypothesis 2 (H2) stated that there would be statistically significant improvements in skin temperature symmetry for the grounded but not for the sham-grounded group. Table 3 shows a comparison of facial right-left symmetry for the grounded and the control group. It was found that the right-left differences of temperature markers decreased for the grounded group (symmetry increased) while the opposite was true for the control group. The difference in symmetry change between groups was statistically significant $(p=0.0284)$. Regarding the torso, the results for change in hot vs. cold spot differences are presented in Table 5. A statistically significant decrease in hot/cold spot difference can be seen for the grounded group $(p=0.0364)$ while a non-significant increase was observed for the control group $(p=0.423)$. The difference in hot/cold spot results between groups is statistically significant $(p=0.0073)$. These results support $\mathrm{H} 2$.

Hypothesis 3 (H3) stated that there would be a significant change in temperature relating to normalization of vascular/lymphatic activity bilaterally of $0.5^{\circ} \mathrm{C}$ or more of the same size parameter/geographical location taking into account inhibiting factors involved with the lymphatic/venous return to the heart including the diaphragm, gallbladder, and scalenus. This hypothesis relates to venous blood/lymph circulation in the torso. A decrease in hot/cold spot has already been documented (H2). There is also a change in abdominal heat pattern that happens only for the grounded group and is an indication of improved fluids circulation and autonomic nervous system regulation in the abdomen as explained in details in the Clinical Observations section below. Figure 6 shows that the average temperature around the umbilicus of subject $\mathrm{A}$ increased by a very significant $2.51^{\circ} \mathrm{C}$. The same region also had a significant increase $\left(0.5^{\circ} \mathrm{C}\right)$ after grounding for subject $\mathrm{B}$ (Figure 9). While the region around the umbilicus did not increase significantly for subject $C$ (Figure 12), the pattern changed in a manner that is unique to grounded subjects (see discussion in the Clinical Observations section below) and there is an increase in temperature of the cool spot just above the umbilicus by a significant $1.25^{\circ} \mathrm{C}$. Coupling these results for grounded subjects with significant decreases around the umbilicus for 2 control subjects $\left(2.24^{\circ} \mathrm{C}\right.$ for subject $\mathrm{D}$; $0.89^{\circ} \mathrm{C}$ for subject $\mathrm{E}$ ) and a cooling off of the torso and a significant cooling off of the face by $0.71^{\circ} \mathrm{C}$ for subject $\mathrm{F}$, it is concluded that $\mathrm{H} 3$ is supported.

Finally, Hypothesis 4 (H4) stated that there would be normalization (improved symmetry) about pain site parameter evaluation designated with a Pointer View (PV) performed by the subject. Subject A has a history of extensive pain indicated at a 3 on a scale from 1 - 10 when she came in for the session. Most of the entire neck/ back/arms/forearms/thighs/legs dropped down to a 1 level after the grounding session. Subject B has a history of right thumb pain with surgery in the region and right lateral breast region pain of a mild intensity. She reported that the pain in the region of the right breast disappeared after the session. Subject $C$ has a history of 2 fibroids in the uterus with intermittent lower pelvic pain ranging from $2-4$, also pain in the right lower pelvic region. Her abdominal pain disappeared after the grounding session. All 3 grounded subjects showed a significant decrease in hot/cold abdominal spot temperature difference while it increased significantly for two control subjects and did not change for the third control subject. It is concluded that $\mathrm{H} 4$ was supported.

\subsection{Clinical Observations}

Upon examination, pre-post grounded subjects overall improved symmetry was consistently observed. While restoring proper autonomic function, yet sustaining core temperature, grounded subjects appear to take on a number of changes in skin temperatures of different parts of the body. Without external intervention, skin temperatures, once established, are consistent year in and year out. This fact suggests that there is a thermal thumbprint of the physiological state of an individual. We may be discovering that a person's thermal pattern does not change over time unless there has been a significant change in the physiological characteristics of that region for 
that individual. The investigators of this study recognize that thermography as a diagnostic assessment tool has been widely misunderstood and criticized because of the lack of ability to "norm" across groups. This study helps to clear up some of these misunderstandings by assessing the unique patterns, symmetry and temperature variances for an individual. Where sustained changes in abdominal temperature patterns usually take weeks to months to show with most interventions, temperature patterns of people who were grounded changed in minutes. All grounded subjects had an increase in total abdominal/small intestine average temperature, while all sham-grounded subjects had a decrease in average abdominal temperature.

Additionally, grounded subjects showed significant changes in the characteristics of the shape of gut distension sites, see for example subject $\mathrm{C}$ in Figure 22. These changes in abdominal thermal characteristics of shape/ location in the grounded subjects are indicative of digestive fluid motility related to improved autonomic function. The abdomen of these subjects appears more homogeneous suggestive of improved symmetry even though the bilateral thermal evaluation was not performed in this region.

One of our clinical findings over the years using thermal imaging and Earthing is that grounding produces a surge in vascularity in the extremities, over the hiatal region of the stomach and the supraclavicular regions. These surges appear to be temporary autonomic responses toward homeostasis. Figure 23 shows an example of improved vascularity of the extremities and Figure 24 presents an example for the hiatal region of the stomach and the supraclavicular regions.

Next, an example to illustrate the differences between Earthing and artificial methods for improving return of blood and lymph fluid to the heart is presented. Compare the just mentioned results to the example of a pre-post thermal study formed on a subject with a cardiac system device (Figure 25). The device compressed feet and legs for 1 hour in a synchronized pattern beginning with the feet to forward the blood and lymph to the abdomen. The process was performed to improve the venous/lymph return to the heart for cardiac patients. However, significant increased lymphatic/venous congestion below the diaphragm and the head/neck can be seen after the hour of synchronized compression of the lower extremities. There is a suspicious extensive lymphatic involvement with focal spotted heat pattern over the abdomen in this subject. The lymphatic/venous fluids forced out of the legs have yet to get to the heart but for that to happen these fluids need to pass through the diaphragm for a healthy venous flow, which did not happen in this case.

As another example, full body vibration (Evolution Professional Whole Body Vibration Trainer, http://www.evolutionhealth.com/whole-body-vibration/dkn-xg10-pro-whole-body-vibration-trainer/) was tested on 30 subjects with 10 minutes of vibration for the purpose of improving lymphatic/venous congestion [22]. Pre and post thermal images were recorded. It was found that $70 \%$ of the subjects responded significantly with overall reduction in head/neck/abdominal lymphatic/venous congestion thermal markers. Figure 26(a) shows an example of one subject who responded appropriately while Figure 26(b) shows an example of a subject who did not benefit. The subject in Figure 26(b) is one of the 30\% who had increased lymphatic/venous congestion involving improper function related to diaphragm as can be seen from the thermally imaged red areas on the chest/shoulders and around the neck. One of these nonresponsive subjects had a gallbladder attack the night after her first vibration session. She knew she had gallstones but no one had any idea that vibration-increased lymph and venous returns would produce such an adverse response.

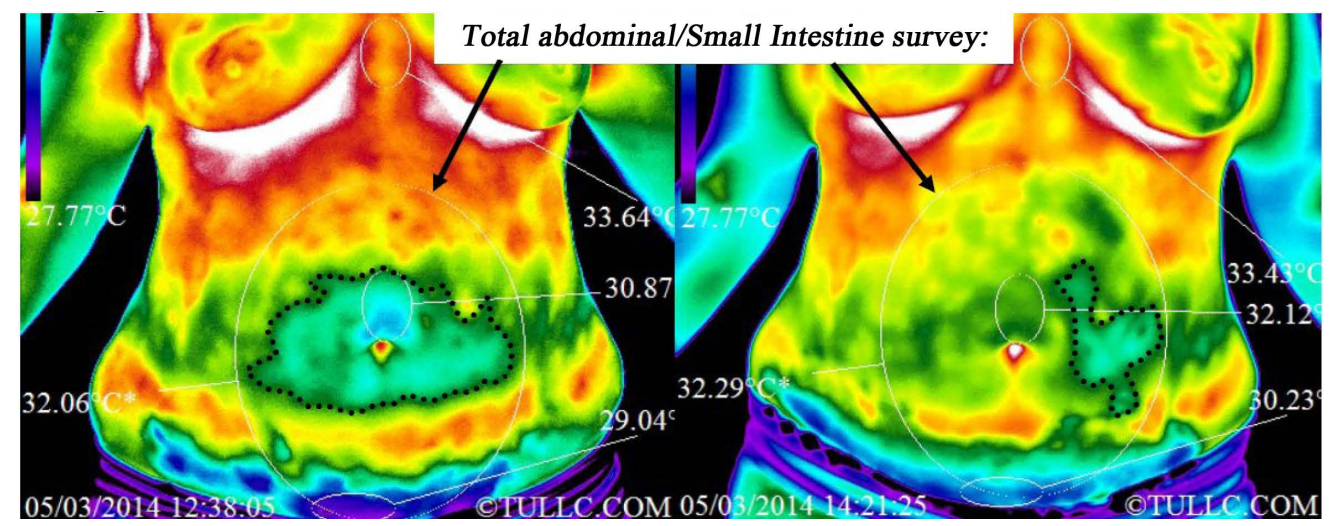

Figure 22. Subject $C$ thermal images showing the cool area around the umbilicus moving to the left and changing shape after one hour grounding session. 


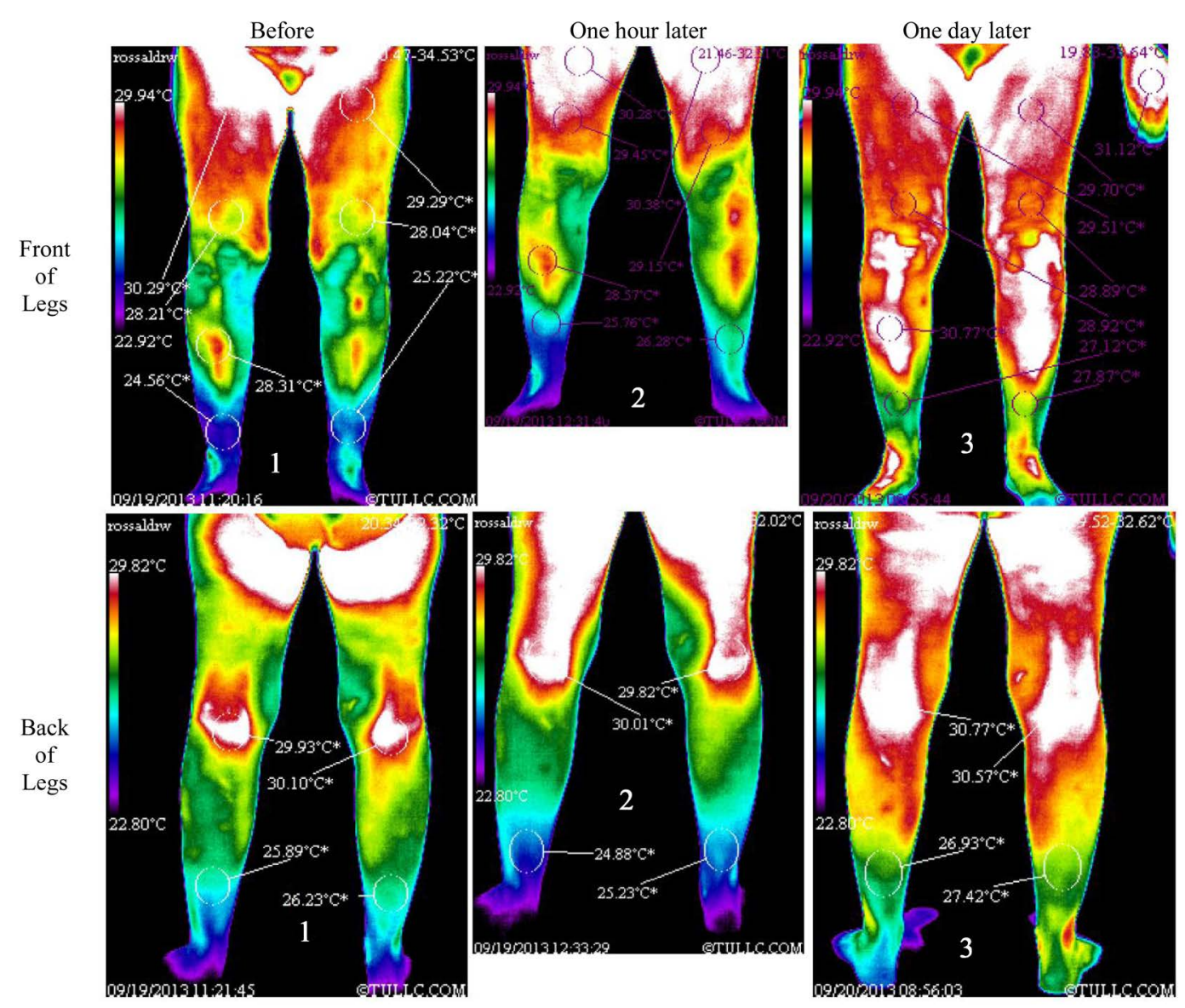

Figure 23. Comparative thermal images before (1), after one hour (2) and after one day (3) of grounding resulting in significant increased vascularity of the lower extremities.

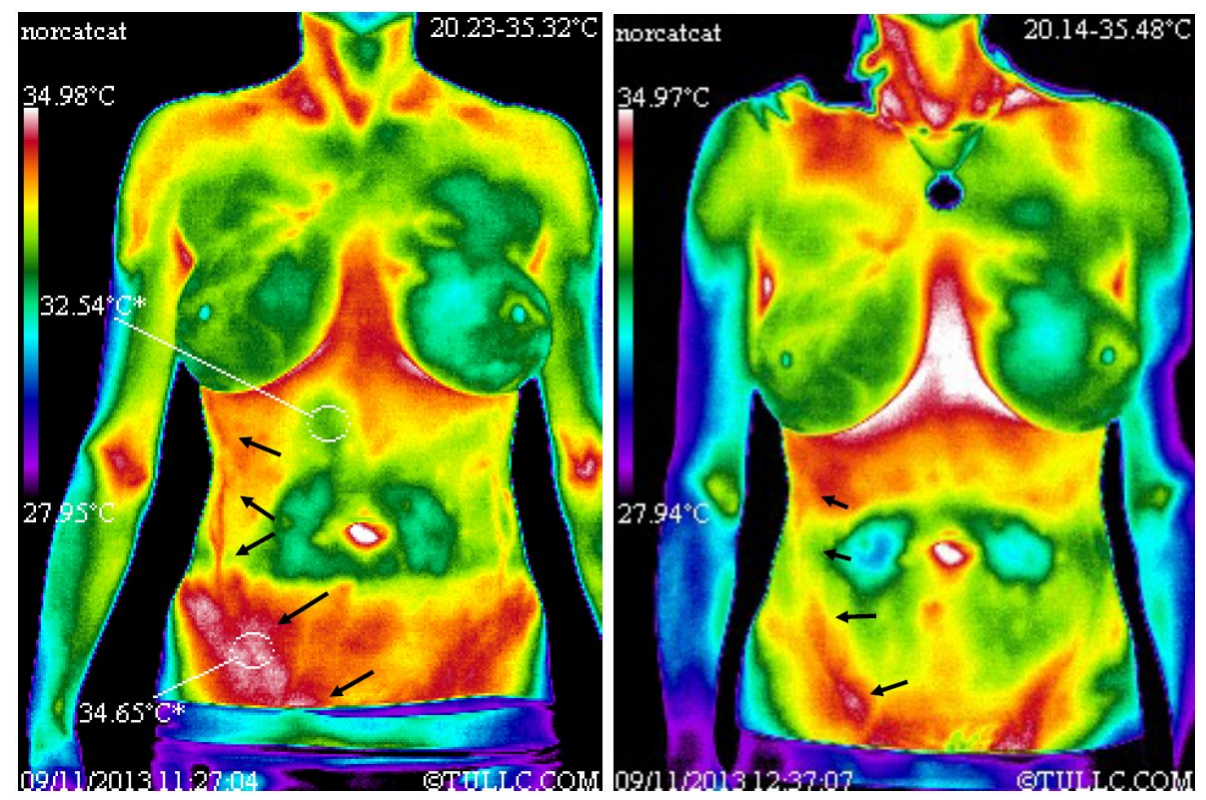

Figure 24. Comparative thermal after 20 minutes of grounding with significant reduction in lower pelvic lymphatic/venous congestion, yet increased over the diaphragm and the base of the neck. These findings are consistent with acceleration of blood and lymph return to the heart. 


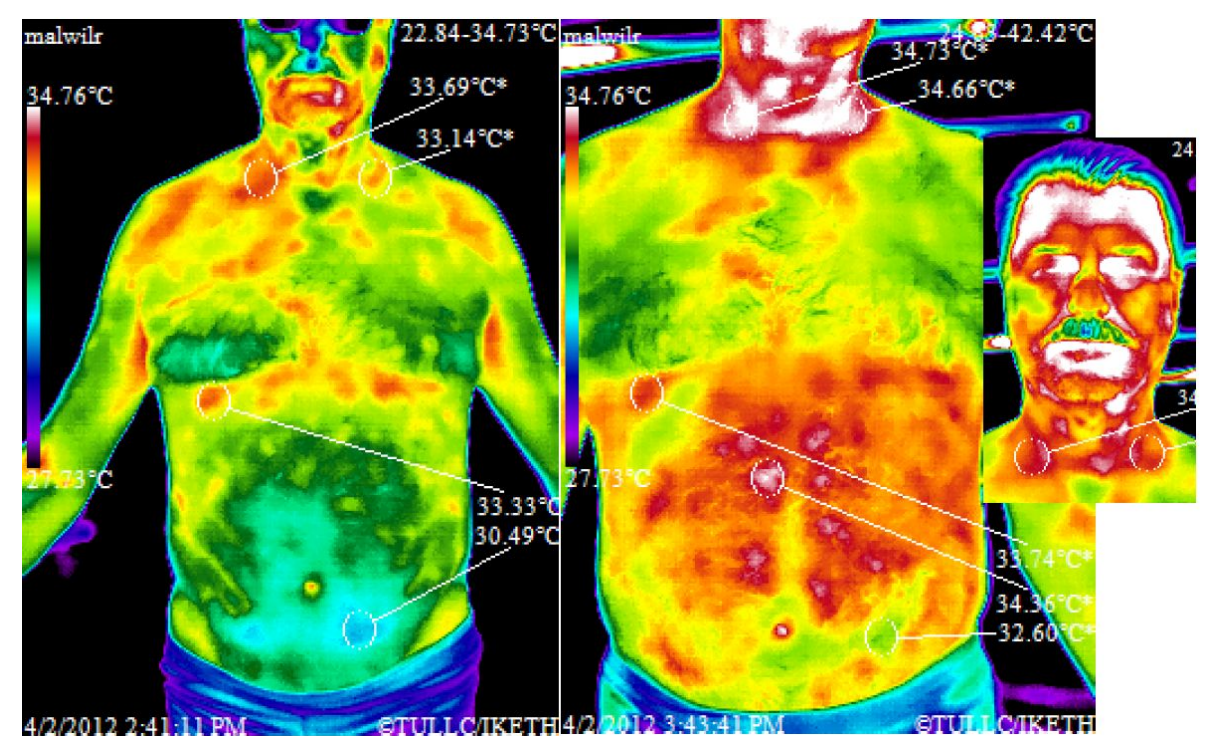

Figure 25. Pre-post thermal study with a cardiac system device compressing feet and legs in a synchronized pattern for one hour to forward blood and lymph to the abdomen.
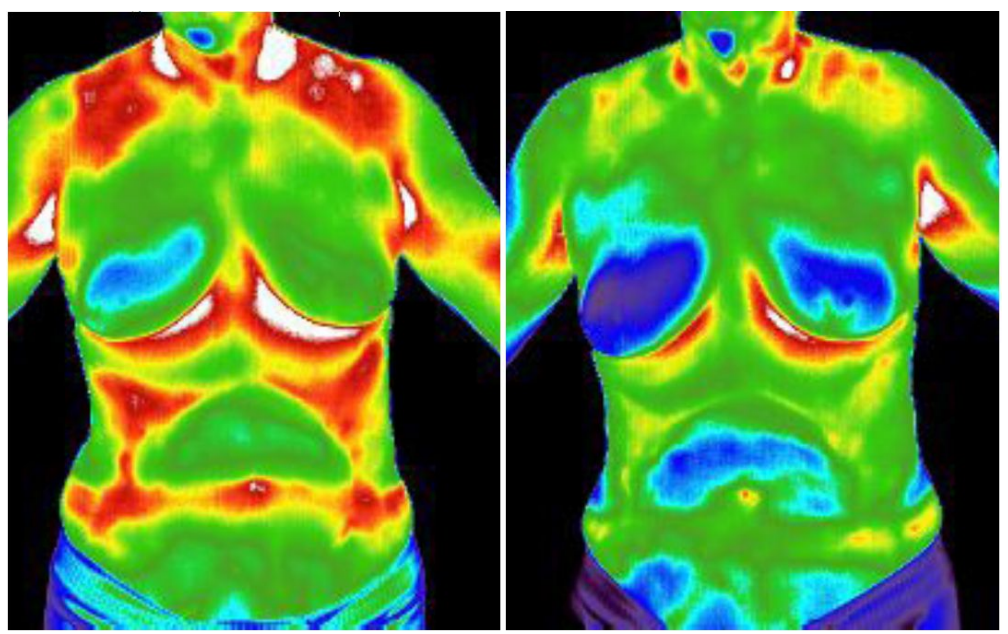

(a)
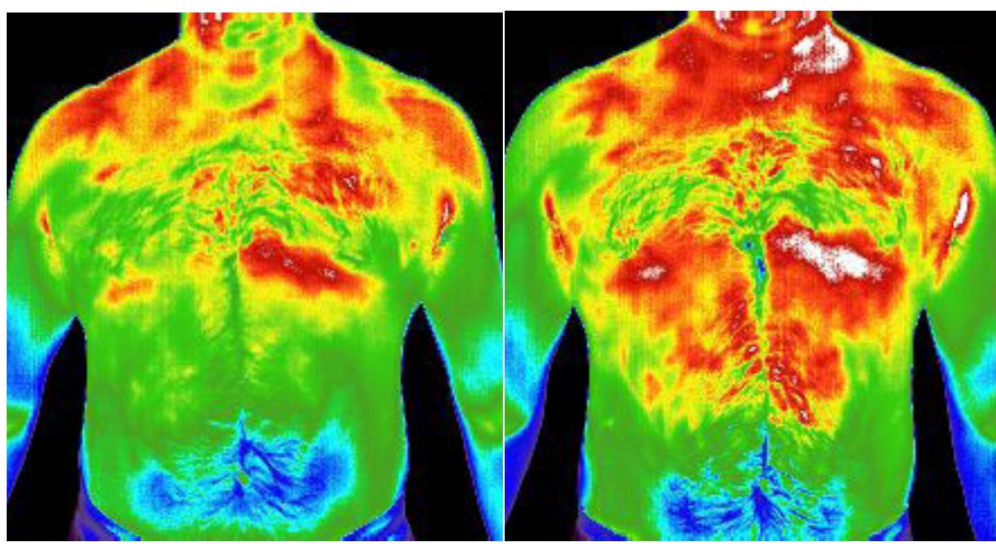

(b)

Figure 26. Pre-post 10 minutes of full body vibration. (a) Case that responded well; (b) Case that did not respond well. 
These examples where it is attempted to mechanically force blood circulation through the diaphragm and their mixed results demonstrate that mechanical methods of increasing vascular return to the heart do not compare with Earthing which improves naturally blood and lymph return to the heart through the diaphragm barrier by having an influence over the ANS and the hypothalamus resulting in improved circulation to the heart and other organs.

The regions of primary routing for lymphatic/venous return to the heart are through the abdominal/thoracic barriers such as the mesentery, peritoneum, and pericardial regions. When restricted diaphragmatic movement occurs, the venous blood returns via the inferior vena cava to the right atrium because it has difficulty returning to the heart using the usual path, causing a rerouting of the venous blood [14]-[17]. In that case, the venous return goes to the outer abdominal wall along the chest to the axilla then to the subclavian vein/superior vena cava then finally to the heart, resulting in a characteristic heat pattern seen over the chest, shoulder and neck areas.

Diaphragmatic myofascial activity is associated with restricted respiration secondary to lack of proper innervation by the phrenic nerve originating from the lower neck (vertebra C4, C5 and C6). Diaphragm disorders may be one sided (the right phrenic nerve controls the right side of the diaphragm, the left phrenic nerve controls left side of the diaphragm). A fully functional diaphragm facilitates proper digestion and proper return of venous/ lymph from the lower body. The dome-like muscle that is the diaphragm primes the venous/lymph fluids back to the heart for two primary goals: oxygenation and proper volume of fluid return to the heart. Improper fluid return to the heart may result in increased blood pressure resulting in venous distention (bulge) of the venous system commonly referred to as hemorrhoid/varicose veins/lower extremity edema.

A functional/coordinated diaphragm with solar plexus stimulation will initiate proper digestive sequence, particularly related to hydrochloric acid (HCL)/pancreatic enzyme/bile delivery from the gallbladder. The proper forwarding of digestive fluids proceeds as follows: from the stomach (mixed with HCL), to the small intestines to neutralize HCL and oil/fat breakdown with bile, and pancreatic enzymes for carbohydrate breakdown and absorption of protein/fats/carbohydrates, to the large intestines for the re-absorption of fluids with proper cultivated bacteria processing feces to produce vitamins. This sequence is predicated by proper valve coordinating such as: pyloric valve (stomach), ileocecal valve (small to large intestines), and anal valve (rectum). Dysfunctional diaphragm and valves may produce the following symptoms: a spastic pyloric valve can result in stomach ulcers, hiatal hernia, acid reflex, and gastroesophageal reflux disease (GERD); a dysfunctional ileocecal valve may result in appendicitis, leaky gut syndrome; and a spastic anal valve can produce hemorrhoids, fistulas, constipation, and toxemia. Note that the gallbladder produces 5 - 10 fold concentrate of bile salts equivalent to 12 hours of liver bile secretion.

Any prolonged presence of digestive fluids, and circulating undigested proteins can result in inflammation/distention (bulge) in the abdomen thus resulting in improper digestion. Digestive disorders lead into food sensitivity and liver defensive responses causing frequent histamine/allergen responses that thermographically are noted as increased temperature around the eyes/nose/mouth. Diaphragm disorders can result in increased upper abdominal temperatures suggestive of venous/lymphatic congestion due to stasis (prolonged presence) of fluids having difficulty retuning to the inferior vena cava through the diaphragm thus forcing fluids to be routed to the exterior abdominal walls up the chest into the subclavian vein/superior vena cava and then to the heart. The process just described was termed by Dale Alexander the Inside-Out Paradigm [15]-[17]. Chronic diaphragm dysfunction with emotional distress may lead into gallbladder disorders causing fluids left in the gallbladder for prolonged periods that can result in stone development. A distended gallbladder with gallstones lies in front of the inferior vena cava (the primary route for the return venous fluid from the lower body) causing entrapment significantly adding to the Inside-Out Paradigm as described.

The integrity of the spinal/pelvic structure influences digestion due to the possible lack of proper innervation and physical abdominal compression. The spinal/cranial/pelvic structural unit responds much like a door that when stress out of normal level distorts (like a warped door). Spinal distortion, especially in the sitting posture, tends to displace the organs adding abdominal pressure when the pelvic is backwardly tilted producing a flattened to reversed lower back curve. The distance between the pubic bone to the sternum may decrease $50 \%$ from sitting to standing resulting in significant abdominal internal pressure producing the following effects: restricted diaphragm motion, difficulty in venous/lymph movement including difficulty in venous/lymph fluids from the lower extremities to return to the heart. Left/right lateral (side to side) spinal displacement (leaning) may add more difficulty over the side of abdominal clenching adding to the lack of venous/lymphatic fluid draining.

Lymph derived from the liver and intestines carry nutrients, fats, and also bacteria and large particles which 
can include endotoxins/exotoxins from those organisms found in the gut. Thus, lymph nodes are used as collection sites for bacteria and toxins. When these sites are overwhelmed these bacteria/toxins can overflow in the arterial system resulting in cardiovascular problems [23].

\subsection{Statistical Analyses}

In Table 1, it can be seen that the average difference in temperatures of the front view of the face decreased after both the grounded and sham-grounded sessions ( $p=0.0423$ and $p=0.00008$, respectively). However, the decrease in temperature was more than $0.5^{\circ} \mathrm{C}$ for the sham grounded group only $\left(0.64^{\circ} \mathrm{C}\right.$ vs. $0.25^{\circ} \mathrm{C}$ for the grounded group). It is also noted that there was a statistically significant difference between groups $(p=0.002)$, indicating that the temperature decrease of the sham-grounded group is statistically significantly larger than that of the grounded group. Subject A was the only subject with an increase in temperature of the face and the increase was significant (greater than $0.5^{\circ} \mathrm{C}$ ) and as such is the main contributor of the reason why the temperature decrease of the grounded group was less than that of the control group. However, subject A was chosen because it represented other subjects with similar increases after a grounding session. This was not seen for the control group i.e. no control subject showed an increase in facial temperature after a sham-grounding session. In this regard, the results of Table 1 are a reflection of the trends seen in our grounded subjects compared to the control subjects as a whole. In summary, Table 1 results indicate that, in average, the front of the face cooled a little for the grounded group while it cooled a lot more for the control group, in fact so much more that it can be said that the cooling seen for the control group is significantly different from that of the grounded group.

Table 2 shows a decrease in the average temperature of the left and right sides of the face combined. The decrease was negligible for the grounded group $\left(0.04^{\circ} \mathrm{C} ; p=0.878\right)$ while it was significant for the control group $\left(0.42^{\circ} \mathrm{C} ; p=0.00256\right)$. There was a statistically significant difference in temperature decreases between groups ( $p=0.0017$ ), indicating that the temperature decrease of the sham-grounded group can be considered significant while that of the grounded group is not. The minimal changes in facial temperatures seen in Table 1 and Table 2 for the grounded group are an indication of higher blood flow to the face for that group.

Table 3 shows decreased left-right difference in facial temperature markers for the grounded group (an average of $\left.-0.10^{\circ} \mathrm{C}\right)$ and an increased for the control group $\left(+0.19^{\circ} \mathrm{C}\right)$. This difference is statistically significant $(p=$ 0.0284). In other words, facial temperature symmetry improved for the grounded group but became worse for the control group and these group differences in symmetry are statistically significant i.e. there is a real improvement in symmetry for the grounded group compared to the control group. The more uniform temperature of the different regions of the face for the grounded group is an indication that there is better blood flow as compared to the sham-grounded (control) group.

A comparison of the change in maximum and minimum temperature differences of the abdomen (Table 4, rows named “Abd-Hot-Cold:”) shows that the average max-min temperature difference decreased by a significant $1.41^{\circ} \mathrm{C}$ after the grounding session while it increases by $0.47^{\circ} \mathrm{C}$ after the sham-grounded session, a significant difference of $1.88^{\circ} \mathrm{C}$. This is an indication of improve blood/lymph/fluids circulation in the abdomen for the grounded group. Table 5 presents a more detailed analysis of these hot-cold spots. From that table, it is seen that the decrease in average hot-cold spot temperature differences for the grounded subjects is statistically significant ( $p=0.0364$ ) while the increase in average hot-cold spot temperature differences for the control group is not statistically significant $(p=0.423)$. Furthermore, the difference in hot-colds spots differences behavior between groups was statistically significant $(p=0.0073)$. In other words, there was a real decrease in hot-cold spots temperature differences for the grounded group but no real change for the control group and the difference in hot-cold spots temperature differences between group was real, i.e., caused by some factor (grounding), not placebo.

Coming back to Table 4, the average of the temperature markers around the umbilicus, between breasts, abdominal cold spot and abdominal hot spot, shows a significant increase by $0.88^{\circ} \mathrm{C}$ for the grounded group $(p=$ 0.0631; not statistically significant but the temperature increased by more than $0.5^{\circ} \mathrm{C}$ ) while the average of the temperature markers for the same spots for the sham-grounded group show a statistically significant decrease of $1.20^{\circ} \mathrm{C}(p=0.0200)$, for a temperature difference of $2.08^{\circ} \mathrm{C}$ between groups. These results point toward very significant differences in the physiological responses of the grounded subjects compared to the sham-grounded (control) subjects. As stated in the Clinical Observation section of the Discussion, abdominal temperature patterns rarely change unless there is an influencing factor (in this case grounding). It can be seen from Figure 15, 
Figure 18 and Figure 21 that there is a drop in temperature of the abdomen for all 3 sham-grounded subjects (even while the max-min difference increased) and that the abdomen retained the same temperature patterns before and after sham-grounding (the regions with low temperatures stayed cool or became cooler and the warm regions remained warm regions even if cooler or became warmer). This is not the case for grounded subjects where the changes in abdominal temperature increased or decreased in different ways in different regions. For example, Figure 6 shows that almost the entire abdomen of subject A became very hot below the breasts and above the waist line after the grounding session, most of the cool spots around the umbilicus became hot spots; Figure 9 shows a cool region around the umbilicus of subject B that became a hot region on the left of the umbilicus; Figure 12 shows a cold region around the umbilicus of subject $C$ which moved to the left of the umbilicus after the grounding session (taking the shape of a duck). These distinct changes in temperature pattern are only seen for the grounded subjects and may be explained by a change in fluids movements and blood circulation that took place only for the grounded group.

Combining face and torso statistics (Table 6) yields similar results in that there is minimal change in the overall average temperature for the grounded group (an increase by $0.06^{\circ} \mathrm{C}$ ) and a significant decrease in overall average temperature for the sham-grounded group by $0.64^{\circ} \mathrm{C}(p=0.00005)$. These differences in temperature behaviors between groups is not due to chance alone, they are very significant $(p=0.00001)$, and consequently must be attributed to a factor different than placebo i.e. grounding.

Low temperature patterns reflect distensions in the abdomen and these distensions improved (decreased) for grounded subjects but not for the sham-grounded control subjects. This result reflects changes in fluid movement patterns in the small intestine or the colon and this is seen only in the grounded group. This result suggests improvement in digestive fluid movements, which denotes an improvement in autonomic nervous function and regulation. This result is congruent with the results obtained using the LSCI where improvement in ANS control of the facial blood circulation was found [7].

\subsection{Possible Mechanisms}

The results presented here show that even one-hour contact with the Earth appears to promote autonomic nervous system (ANS) control of body fluids and peripheral blood flow that may improve blood circulation in the torso and face, facial tissue repair, skin health and vitality and optimize facial appearance. All grounded subjects showed changes in abdominal patterns not seen with control subjects as already noted (Figure 22). This is an indication of intervention effects (grounding). The lack of autonomic control tends to produce consistent markers and patterns in the same geographical regions of the abdomen. Significant increase in vascular fluidity, digestive fluids and cerebral spinal fluid with improved autonomic control is related intestinal lining motility. As already noted, clinical observations over the years have shown repeatedly that these changes in pattern do not happen without an external intervention.

The intestinal blood circulation and peristaltism are controlled by many local and neuronal mechanisms. However, these control systems are modulated by the ANS [24] [25]. Therefore, the present results suggest that connection with the Earth supports a more efficient autonomic nervous system (ANS) regulation of the abdomen as well as of the face. It is also possible that the lower viscosity and aggregation of red blood cells (RBCs) produced by grounding result in an improved blood flow in the face and abdomen [7] [26]. In a previous publication, grounding was shown to improve regulation of facial blood flow as shown by dynamical pictures taken with a laser speckle contrast imaging (LSCI) camera [7]. Since this previous finding came from the same study with the same subjects, the discussion and mechanisms mentioned in [7] also apply here; namely, increased zeta potential, decreased red blood cell aggregation, improved overall physiology, and reduction in stress [7].

\subsection{Study Limitations}

The results shown here for six subjects are representative of what was seen with the other subjects of this study. The results of all 40 subjects could not be presented for lack of space but they are similar to the results presented here. Because of the small number of subjects, this experiment needs to be replicated and subjects could be followed for longer period of times to confirm improvements in blood circulation of the face and abdomen as well as improvement in digestive and intestinal function. More robust statistical analyses could be performed with more subjects. Limitation in resources prevented the authors to perform statistical analyzes on all the subjects.

The present results reinforced the previous observation that the ANS increased its control of the body for the 
grounded subjects but no real change was seen for the controls except changes that can be explained by relaxation. The different ways of looking at temperature marker changes before vs. after sessions and the different comparisons between groups confirm that there is enough difference between the grounded group and the shamgrounded (control) group to suggest a beneficial effect of grounding i.e., mainly an increase ANS control over the distribution of fluids in the body including better blood flow to the face after a one hour grounding session. Only data of 3 subjects per group have been presented but these subjects are representative of 27 grounded subjects and the 13 sham-grounded subjects for which thermal images have similarly been recorded.

\section{Conclusion}

The Earth possesses a form of easily accessible beneficial natural energy that has been demonstrated previously to influence positively human physiology and health. The results of this innovative study demonstrate, for the first time, that even one-hour contact with the surface of the earth appears to promote significant increase in blood flow regulation to the head and torso that may enhance skin tissue repair, health, and vitality, and optimize facial appearance. Thermal imaging showed clearly improved fluids movements in the abdomen as well as improved blood circulation of the face and throughout the torso, which in turn, may translate into better health. Further study, using larger comparison groups and longer follow up time, is warranted in order to confirm the novel influence of the Earth as a protector of skin and overall health and wellbeing.

\section{Acknowledgements}

The authors wish to thank Linda Hayes, C.C.T., and Theresa Williams, C.C.T., of TTI for recruiting study subjects, conducting all imaging activities, as well as administering subject questionnaires. Earth FX, Inc., funded the study and the products were donated by earthing.com.

\section{References}

[1] Ober, C., Sinatra, S.T. and Zucker, M. (2014) Earthing: The Most Important Health Discovery Ever! 2nd Edition, Basic Health Publications, Laguna Beach.

[2] Williams, E.R. and Heckman, S.J. (1993) The Local Diurnal Variation of Cloud Electrification and the Global Diurnal Variation of Negative Charge on the Earth. Journal of Geophysical Research, 98, 5221-5234. http://dx.doi.org/10.1029/92JD02642

[3] Anisimov, S.V., Mareev, E.A. and Bakastov, S.S. (1999) On the Generation and Evolution of Aeroelectric Structures in the Surface Layer. Journal of Geophysical Research, 104, 14359-14367. http://dx.doi.org/10.1029/1999JD900117

[4] Oschman, J.L. (2007) Can Electrons Act as Antioxidants? A Review and Commentary. Journal of Alternative and Complementary Medicine, 13, 955-967. http://online.liebertpub.com/doi/pdfplus/10.1089/acm.2007.7048 http://dx.doi.org/10.1089/acm.2007.7048

[5] Oschman, J.L. (2009) Charge Transfer in the Living Matrix. Journal of Bodywork and Movement Therapies, 13, 215228. http://dx.doi.org/10.1016/j.jbmt.2008.06.005

[6] Chevalier, G., Sinatra, S.T., Oschman, J.L., Sokal, K. and Sokal, P. (2012) Earthing: Health Implications of Reconnecting the Human Body to the Earth's Surface Electrons. Journal of Environmental and Public Health, 2012, Article ID: 291541. http://dx.doi.org/10.1155/2012/291541

[7] Chevalier, G. (2014) Grounding the Body Improves Facial Blood Flow Regulation: Results of a Randomized, Placebo Controlled Pilot Study. Journal of Cosmetics, Dermatological Sciences and Applications, 4, 293-308. http://dx.doi.org/10.4236/jcdsa.2014.45039

[8] Chevalier, G. (2015) The Effect of Grounding the Human Body on Mood. Psychological Reports, 116, 534-542. http://dx.doi.org/10.2466/06.PR0.116k21w5

[9] Berz, R. and Sauer, H. (2007) The Medical Use of Infrared-Thermography-History and Recent Applications. Thermografie-Kolloquium 2007 by DGZfP, Stuttgart, 1-12.

[10] Ring, E.F.J. (2004) The Historical Development of Thermal Imaging in Medicine. Rheumatology, 43, 800-802. http://dx.doi.org/10.1093/rheumatology/keg009

[11] Uematsu, S., Edwin, D.H., Jankel, W.R., Kozikowski, J. and Trattner, M. (1988) Quantification of Thermal Asymmetry. Journal of Neurosurgery, 69, 552-555. http://dx.doi.org/10.3171/jns.1988.69.4.0552

[12] Niu, H.-H., Lui, P.-W., Hu, J.S., Ting, C.-K., Yin, Y.-C., Lo, Y.-L., Lui, L. and Lee, T.-Y. (2001) Thermal Symmetry of Skin Temperature: Normative Data of Normal Subjects in Taiwan. Chinese Medical Journal (Taipei), 64, 459-468. 
[13] Sherman, R.A., Karstetter, K.W., Damiano, M. and Evans, C.B. (1994) Stability of Temperature Asymmetries in Reflex Sympathetic Dystrophy over Time and Changes in Pain. The Clinical Journal of Pain, 10, 71-77. http://dx.doi.org/10.1097/00002508-199403000-00010

[14] Guyton, A.C. (1971) Textbook of Medical Physiology Fourth Edition: Regulation of Body Temperature-Function of the Hypothamalus. Saunders, 837-840.

[15] Alexander, D.G. (2004) Healing From the Core. Massage Today. http://www.dale-alexander.com/HealingCore.html

[16] Alexander, D.G. (2005) Equalizing the Pressure. Massage Today. http://www.dale-alexander.com/EqualizingPressure.html

[17] Alexander, D.G. (2005) Survival vs. Quality of Life. Massage Today. http://www.dale-alexander.com/Survival.html

[18] Venturi, S. and Venturi, M. (2009) Iodine in Evolution of Salivary Glands and Oral Health. Nutrition and Health, 20, 119-134. http://dx.doi.org/10.1177/026010600902000204

[19] Pauling, J.D., Shipley, J.A., Raper, S., Watson, M.L., Ward, S.G., Harris, N.D. and McHugh, N.J. (2012) Comparison of Infrared Thermography and Laser Speckle Contrast Imaging for the Dynamic Assessment of Digital Microvascular Function. Microvascular Research, 83, 162-167. http://dx.doi.org/10.1016/j.mvr.2011.06.012

[20] Heesch, C.M. (1999) Reflexes That Control Cardiovascular Function. American Journal of Physiology-Advances in Physiology Education, 22, S234-S243.

[21] Landesberg, G., Adam, D. and Akselrod, S. (1996) Very Slow Response of the Baroreflex Regulation System: A Tool for Understanding Cardiovascular Control. Computers in Cardiology, 23, 161-164. http://dx.doi.org/10.1109/cic.1996.542498

[22] Total Thermal Imaging, in House Clinical Trial, 2012, Unpublished.

[23] Manco, M., Putignani, L. and Bottazo, G.F. (2010) Gut Microbiota, Lipopolysaccharides, and Innate Immunity in the Pathogenesis of Obesity and Cardiovascular Risk. Endocrine Reviews, 31, 817-844. http://dx.doi.org/10.1210/er.2009-0030

[24] Rosenblum, J.D., Boyle, C.M. and Schwartz, L.B. (1997) The Mesenteric Circulation. Anatomy and Physiology. Surgical Clinics of North America, 77, 289-306. http://dx.doi.org/10.1016/S0039-6109(05)70549-1

[25] Fitzsimmons, L. and Hadley, S.A. (1990) Neural Mechanisms and Patterns of Abdominal Pain. Topics in Emergency Medicine, 12, 1-8.

[26] Chevalier, G., Sinatra, S.T., Oschman, J.L. and Delany, R.M. (2013) Earthing (Grounding) the Human Body Reduces Blood Viscosity_A Major Factor in Cardiovascular Disease. Journal of Alternative and Complementary Medicine, 19, 102-110. http://dx.doi.org/10.1089/acm.2011.0820 


\section{Appendix A}
A.1. Thermal Imaging Protocol Prior to Appointment
1) No shaving $2 \mathbf{2 4}$ hours.
2) No sunbathing/ exercising 12 hours (No imaging if sunburned until recovered).
3) No lotions, creams, deodorants, powders 2 hours prior (must be wiped off gently).
4) No eating, no eating or drinking caffeine/ nicotine products 2 hours prior.
5) No manipulation/ massage/ ultrasound therapy $\underline{2}$ hours prior.
6) No mints/gum chewing $\underline{2}$ hours prior.

The thermal imaging technician will verify that each of the 6 points above has been followed. Since each of these points has a clear thermal signature, the technician will be able to determine if one or more of these are present in the thermal images and ask the subject to come back another time if one of these patterns is found.

\section{A.2. Thermal Imaging Protocol during Appointment}

At the beginning and at the end of a session, a series of thermal images will be taken of the subject with the Thermography Unlimited (TU), LLC software. This software has the template images shown in Figure A1 to properly identify each image. Template images 9, 10, 11 and 16 will be used in this study. All template images will have a temperature window span of $7^{\circ} \mathrm{C}$.

Common temperature measurement sites with exactly the same ellipse parameters (markers) will be performed within TU software and are transferable from an initial study file to the comparatives (see Appendix B for ellipse locations or markers to be used for comparisons). Markers render an average temperature able to be placed directly on the image.

\section{Appendix B}

List of temperature markers for quantitative comparison of the average temperature inside one marker with the average temperature inside another marker related by body location or otherwise.

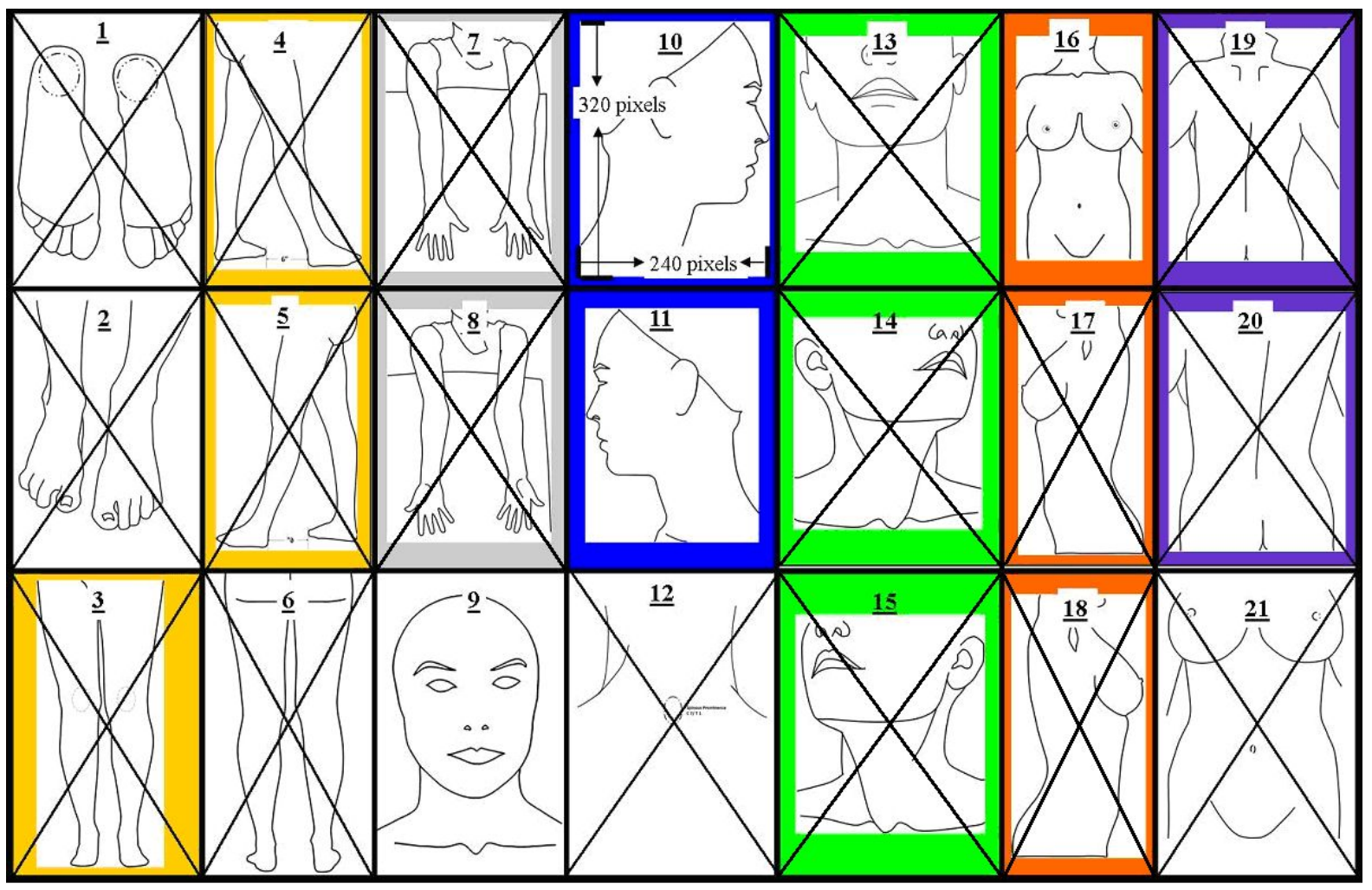

Figure A1. Guide/template images used by TU LLC software. 
- Frontal thermal markers: above the medial canthus on the forehead, associated with suboccipital entrapment of the vertebral artery/greater occipital nerve, dural conflict at the foramen magnum influencing 5th cranial nerve, orbital/ocular distress.

- Orbitopalpebral sulcus superior markers: upper eyelids, classically this region has the highest temperature of the face/neck, while the ethmoid sinus region has the lowest temperature.

- Eye thermal markers: indications of excessive lymphatic/venous congestion/inflammation; may be correlated with kidney distress commonly caused by digestive disorders resulting in kidney waste management issues.

- Maxillary sinus markers: chronic sinus disorders having hypothermia suggestive of chronic sinus dysfunction related to 5th cranial disorder. Acute sinus disorder with hyperthermia (commonly both acute/chronic) will have throat inflammatory temperatures due to sinus drainage.

- Total face thermal maker: used to compare total average temperature of the face.

- Periodontal/oral pathology thermal markers (also termed mouth corner markers): inflammatory responses orally with submandibular spotted lymphatic involvement that can be forwarded into deep/superficial anterior neck nodes usually ending above the thyroid.

- Supraclavicular markers: demonstrates lymphatic/venous congestion responses due to entrapment between over contracted scalene/sternocliedo into the first rib complicated by dropped shoulder and lateral head/neck tilt. The supraclavicular region has significant increases in temperature related to lower body venous return redirected/rerouted into the base of the cervical spine 14 - 16 .

- Temporal mandibular joint marker: above the external auditory meatus.

- Carotid bifurcation marker: below the jaw over the carotid, related to salivary gland when irregular shaped.

- Lateral cervical anterior and posterior markers: survey of myofascial activity.

- Scalene/external jugular vein marker: monitors the contractive state of the scalenus and possible entrapment influences to the external jugular vein/anterior jugular vein. Speculated that carotid inflammation/calcification relate to external jugular vein distention thermal marker due to back pressure unilaterally forwarding that pressure into the carotid artery.

- Lateral face marker: survey symmetry related to 5th/7th cranial nerve autonomic control. Lateral of the face hypothermia may be associated with Bell's Palsy.

- Lower throat thermal marker: related to sinus drainage/stomach regurgitation of stomach fluids.

- Esophageal/hiatal stomach thermal marker: esophageal disorders are commonly involved with 10th cranial nerve disorders which controls the peristaltic lower $1 / 3$ of the esophagus/stomach acidity/pyloric valve opening.

- Abdominal high/low thermal marker: High temperature related to inflammation/improper forwarding of digestive fluids/lymphatic involvement of which aggressive flora development is suspected as cause. Lower temperature related to abdominal distention type thermal marker involving the distal end of the ileum prior to the ileocecal valve.

- Total abdominal/mall intestine survey marker: marker of the extent of distention over the abdomen; marker of the distension of the small intestine if distension is only over the small intestine. Abdominal distention thermal marker patterns generally stay the same, except when changes exist in thermal pattern suggestive of significant change in movement of digestive fluids. Example: Subject C significant low distention site above the umbilicus, while after Earthing resulting in a duck shaped hypothermal left lateral to the umbilicus with increased temperature above the umbilicus by 1.3 degrees $\mathrm{C}$.

- Linear lateral abdominal fold thermal marker: can be used as a barrier to reduce upper to lower abdominal/ pelvic pressure.

- Diaphragm thermal marker: follow linear outline of the diaphragm; well delineated over the superior aspect while more defuse extension of temperature over the inferior aspect depending on the venous congestive responses. Commonly the lateral of most diaphragmtic congestion will coincide with supraclavicular lymphatic/venous congestion. Lower pelvic floor congestion is directly related to diaphragm congestive responses.

Pictorial representations of marker locations 

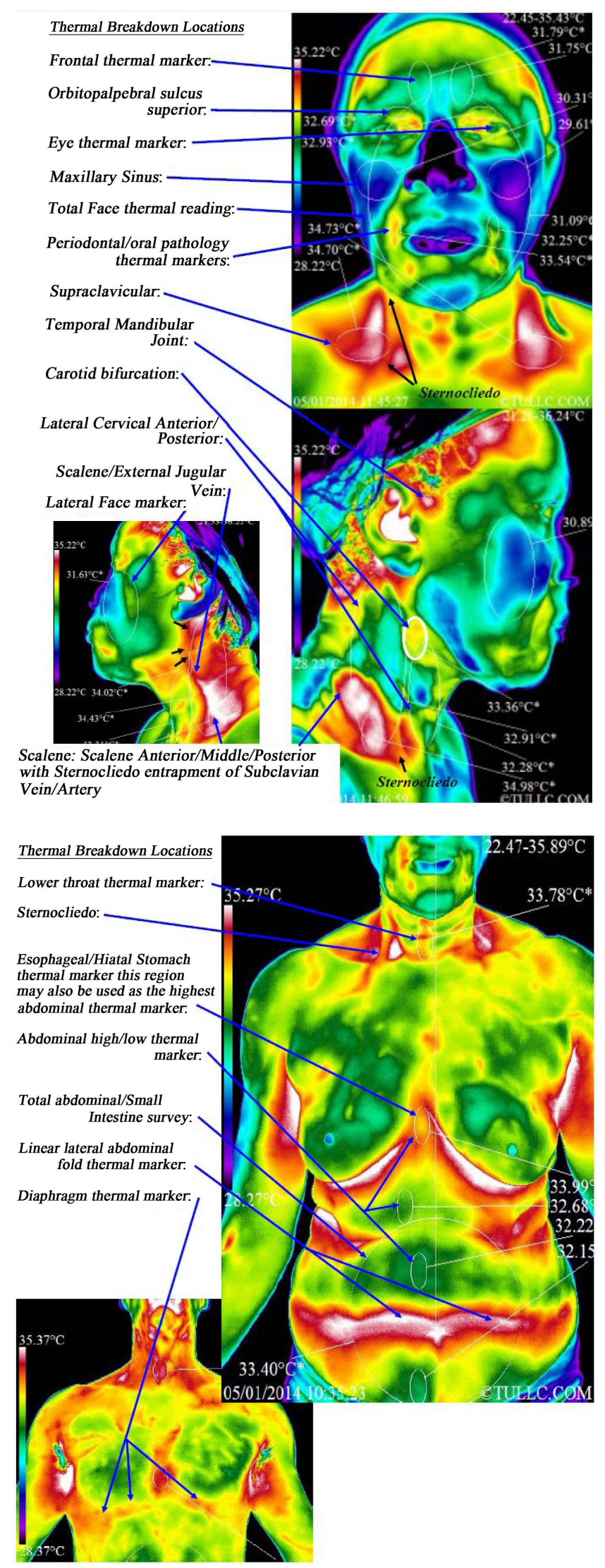


\section{Appendix C}

\section{TTI Wellness Entry Form}

8341 La Mesa Blvd., La Mesa, Ca. 91942 Phone: 619.303.5884

Name: Birth Date:

Mailing Address:

E-Mail:

City: State: Zip:

Emergency Contact: Receive your report: In person / Mail / E-Mail Breast Questionnaire - Occupation:

Home Ph. \# Cell Ph.\# Home Ph.\#

Last Thermogram: / / have you had the following: Diagnosed with breast cancer? Yes / No

If yes: type- Metastatic/Lymphatic node removal/Local When: Diagnosed of other breast disease? Yes / No

Biopsies and your findings? Yes / No

Breast surgery/ implants? Yes /No Mammogram last 12 months? Yes / No

Total mammograms \#

First mammogram \#

Contraceptive over 1 year? Yes / No

Hormone therapy?

Doctors last breast exam?

Monthly breast self exams? Yes / No

Menstrual periods before 12 ? Yes / No

Menstrual stopped after 50 ? Yes $/ \mathbf{N o}$

Total births \#? , age of first born?

Breast symptoms in the last 6 months?

Please demonstrate symptoms with following symbols: "T" for Tendemess; "L" for Lumps

"D/T" for Nipple Dimpling / Thickening.

Change in size "CS"; "NS" Nipple secretion, Biopsy "B"

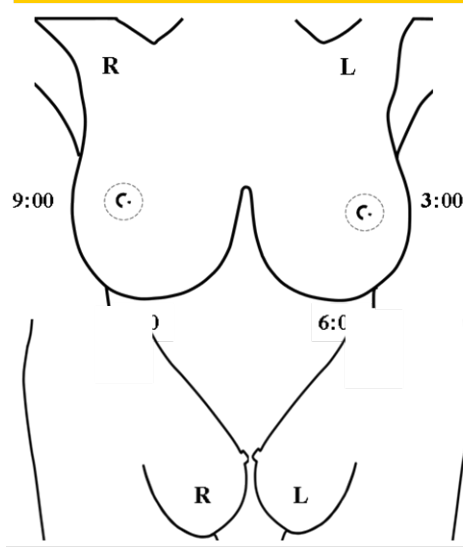

Please demonstrate symptoms with the following symbols with accurate locations on the body figure below: " $\mathrm{N}$ " for numbness; "1-10" for pain 10 being the worst; " $\mathbf{S}$ " for scars; "M" for moles; "F" for fractures; "X" for previous surgeries or current/prior diseases with a line to a brief description.
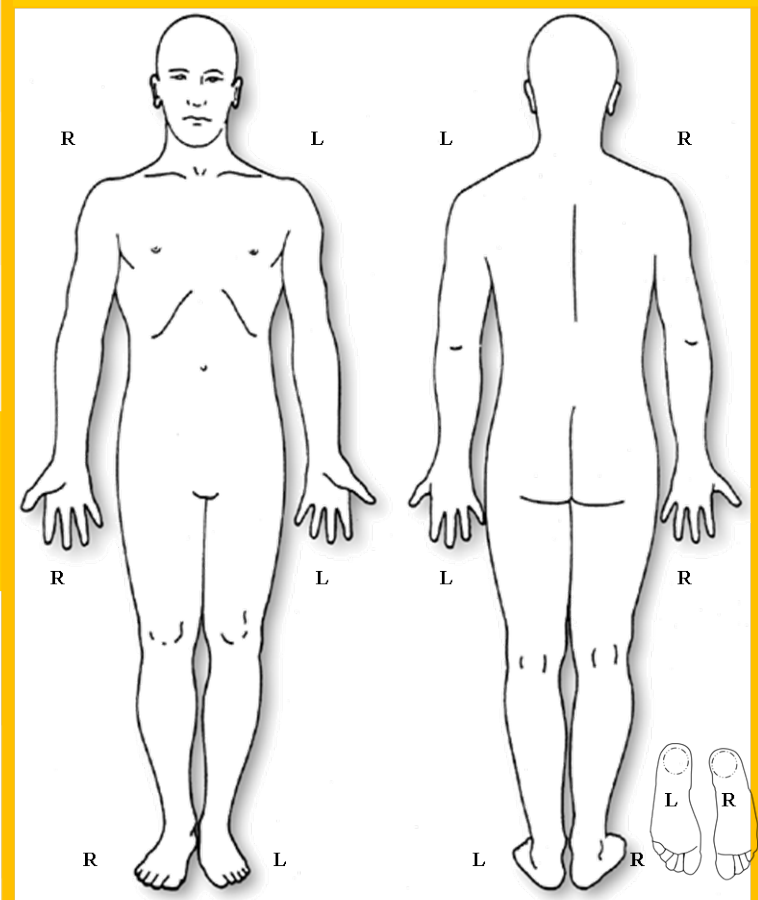

Current Issues /Accidents?

Current medications

\section{Patient Disclosure}

I understand the report generated by my images is intended for use by trained health care providers to assist in evaluation, analysis and treatment. $I$ understand the report is not intended for use by individuals for self-evaluation, diagnosis, or treatment. I understand the report will not tell me whether I have an illness, disease, or other

condition but will be an analysis of the images with respect only to the thermographic findings of the areas discussed in the report. By signing below, I acknowledge and certify that I have read and understand the statements above and consent to the examination. I also authorize the release of information and the receipt of information in the pursuit of comprehensive evaluation and treatment relating to the services provided by Thermography Unlimited, LLC/ Total Thermal Imaging.

Patient Signature:

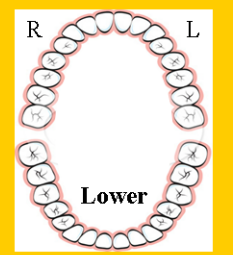
mouth: Root Canal "RC"/Crown "C"/Surgery "S" Mercury Fillings "MF"' "O" = Other For Office Use Only: First Visit 3 month 1 YR Recall Super Bill Description Cost $\$$

Payment Method: Check\# Check / Cash \$

Credit Card \# Visa Exp. Date: Billing Addres

$\begin{array}{ll}\text { MasterCard Discover } & \text { Exp. Date: } \\ \text { SAME AS ABOVE }\end{array}$

